\title{
The nomenclature, definition and classification of cardiac structures in the setting of heterotaxy
}

Jeffrey P. Jacobs, ${ }^{1}$ Robert H. Anderson, ${ }^{2}$ Paul M. Weinberg, ${ }^{3}$ Henry L. Walters III, ${ }^{4}$ Christo I. Tchervenkov, ${ }^{5}$ Danny Del Duca, ${ }^{5}$ Rodney C. G. Franklin, ${ }^{6}$ Vera D. Aiello, ${ }^{7}$ Marie J. Béland, ${ }^{8}$ Steven D. Colan, ${ }^{9}$ J. William Gaynor, ${ }^{10}$ Otto N. Krogmann, ${ }^{11}$ Hiromi Kurosawa, ${ }^{12}$ Bohdan Maruszewski, ${ }^{13}$ Giovanni Stellin, ${ }^{14}$ Martin J. Elliott ${ }^{15}$

${ }^{1}$ The Congenital Heart Institute of Florida, Division of Thoracic and Cardiovascular Surgery, All Children's Hospital/Children's Hospital of Tampa, University of South Florida College of Medicine, Cardiac Surgical Associates, Saint Petersburg and Tampa, Florida, United States of America; ${ }^{2}$ Cardiac Unit, Institute of Child Health, Great Ormond Street Hospital for Children, London, United Kingdom; ${ }^{3}$ Division of Pediatric Cardiology, The Children's Hospital of Philadelphia, University of Pennsylvania School of Medicine, Pennsylvania, United States of America; ${ }^{4}$ Children's Hospital of Michigan, Wayne State University School of Medicine, Detroit, Michigan; ${ }^{5}$ Division of Pediatric Cardiovascular Surgery, The Montreal Children's Hospital of the McGill University Health Centre, Montréal, Quebec, Canada; ${ }^{6}$ Paediatric Cardiology Directorate, Royal Brompton E Harefield NHS Trust, Harefield, Middlesex, United Kingdom; ${ }^{7}$ Heart Institute (InCor), Sao Paulo University School of Medicine, Sao Paulo, Brazil; ${ }^{8}$ Division of Pediatric Cardiology, The Montreal Children's Hospital of the McGill University Health Centre, Montréal, Quebec, Canada; ${ }^{9}$ Department of Cardiology, Children's Hospital, Boston, Massachusetts, United States of America; ${ }^{10}$ Cardiac Surgery, Children's Hospital of Philadelphia, Philadelphia, Pennsylvania, United States of America; ${ }^{11}$ Paediatric Cardiology - CHD, Heart Center Duisburg, Duisburg, Germany;

${ }_{12}^{12}$ Cardiovascular Surgery, Heart Institute of Japan, Tokyo Women's Medical University, Tokyo, Japan;

${ }^{13}$ The Children's Memorial Health Institute, Department of Cardiothoracic Surgery, Warsaw, Poland;

${ }^{14}$ Pediatric Cardiac Surgery Unit, University of Padova Medical School, Padova, Italy; ${ }^{15}$ Cardiac Unit, Great Ormond Street Hospital for Children, London, United Kingdom

Abstract In 2000, The International Nomenclature Committee for Pediatric and Congenital Heart Disease was established. This committee eventually evolved into the International Society for Nomenclature of Paediatric and Congenital Heart Disease. The working component of this international nomenclature society has been The International Working Group for Mapping and Coding of Nomenclatures for Paediatric and Congenital Heart Disease, also known as the Nomenclature Working Group. The Nomenclature Working Group created the International Paediatric and Congenital Cardiac Code, which is available for free download from the internet at [http://www.IPCCC.NET].

In previous publications from the Nomenclature Working Group, unity has been produced by crossmapping separate systems for coding, as for example in the treatment of the functionally univentricular heart, hypoplastic left heart syndrome, or congenitally corrected transposition. In this manuscript, we review the nomenclature, definition, and classification of heterotaxy, also known as the heterotaxy syndrome, placing special emphasis on the philosophical approach taken by both the Bostonian school of segmental notation developed from the teachings of Van Praagh, and the European school of sequential segmental analysis. The Nomenclature Working Group offers the following definition for the term "heterotaxy": "Heterotaxy is synonymous with 'visceral heterotaxy' and 'heterotaxy syndrome'. Heterotaxy is defined as an abnormality where the internal thoraco-abdominal organs demonstrate abnormal arrangement across the left-right axis of

Correspondence to: Jeffrey P. Jacobs, MD, FACS, FACC, FCCP, Cardiovascular and Thoracic Surgeon, The Congenital Heart Institute of Florida (CHIF), Clinical Associate Professor, University of South Florida (USF), Cardiac Surgical Associates (CSA), 603 Seventh Street South, Suite 450, Saint Petersburg, FL 33701. Tel: (727) 822 6666; Cell Phone: (727) 235-3100; Fax: (727) 821 5994; E-mail: JeffJacobs@msn.com, http://www.heartsurgery-csa.com/, http://www.CHIF.us/ 
the body. By convention, heterotaxy does not include patients with either the expected usual or normal arrangement of the internal organs along the left-right axis, also known as 'situs solitus', nor patients with complete mirror-imaged arrangement of the internal organs along the left-right axis also known as 'situs inversus'." "Situs ambiguus is defined as an abnormality in which there are components of situs solitus and situs inversus in the same person. Situs ambiguus, therefore, can be considered to be present when the thoracic and abdominal organs are positioned in such a way with respect to each other as to be not clearly lateralised and thus have neither the usual, or normal, nor the mirror-imaged arrangements."

The heterotaxy syndrome as thus defined is typically associated with complex cardiovascular malformations. Proper description of the heart in patients with this syndrome requires complete description of both the cardiac relations and the junctional connections of the cardiac segments, with documentation of the arrangement of the atrial appendages, the ventricular topology, the nature of the unions of the segments across the atrioventricular and the ventriculoarterial junctions, the infundibular morphologies, and the relationships of the arterial trunks in space. The position of the heart in the chest, and the orientation of the cardiac apex, must also be described separately. Particular attention is required for the venoatrial connections, since these are so often abnormal. The malformations within the heart are then analysed and described separately as for any patient with suspected congenital cardiac disease. The relationship and arrangement of the remaining thoraco-abdominal organs, including the spleen, the lungs, and the intestines, also must be described separately, because, although common patterns of association have been identified, there are frequent exceptions to these common patterns. One of the clinically important implications of heterotaxy syndrome is that splenic abnormalities are common. Investigation of any patient with the cardiac findings associated with heterotaxy, therefore, should include analysis of splenic morphology. The less than perfect association between the state of the spleen and the form of heart disease implies that splenic morphology should be investigated in all forms of heterotaxy, regardless of the type of cardiac disease. The splenic morphology should not be used to stratify the form of disease within the heart, and the form of cardiac disease should not be used to stratify the state of the spleen. Intestinal malrotation is another frequently associated lesion that must be considered. Some advocate that all patients with heterotaxy, especially those with isomerism of the right atrial appendages or asplenia syndrome, should have a barium study to evaluate for intestinal malrotation, given the associated potential morbidity. The cardiac anatomy and associated cardiac malformations, as well as the relationship and arrangement of the remaining thoracoabdominal organs, must be described separately. It is only by utilizing this stepwise and logical progression of analysis that it becomes possible to describe correctly, and to classify properly, patients with heterotaxy.

Keywords: Databases; cardiac relations; cardiac connections; heterotaxy; heterotaxy syndrome; visceral heterotaxy; isomerism; situs ambiguus

I PREVIOUS PUBLICATIONS FROM THE NOMENCLATURE Working Group, unity has been produced by cross-mapping separate systems for coding, as for example in the treatment of the functionally univentricular heart, ${ }^{1}$ hypoplastic left heart syndrome, ${ }^{2}$ or congenitally corrected transposition. ${ }^{3}$ In this manuscript, we review the nomenclature, definition, and classification of heterotaxy, also known as the heterotaxy syndrome, placing special emphasis on the philosophical approach taken by both the Bostonian school of segmental notation developed from the teachings of Van Praagh, ${ }^{4-6}$ and the European school of sequential segmental analysis. ${ }^{7-9}$

It has long been recognised that many of the most complex combinations of cardiac lesions are found in the hearts of patients with jumbled-up abdominal organs, or so-called heterotaxy. It is also well recognised that one of the dominant features of such patients is either absence of the spleen, or presence of multiple spleens on each side of the dorsal mesogastrium. ${ }^{4,10}$ In order to describe properly any complex heart, however, it is essential to provide a description of both the relations of structures within the heart, and the way they are joined together. The first feature accounts for the broad interspatial relations between the various structures. This feature, nonetheless, is not always concordant with the fashion in which two cardiac structures are joined to each other, or in some instances not joined together. ${ }^{11}$ It is the structure of the cardiac components, and their relations, that forms the basis of the segmental approach of Van Praagh and Vlad. ${ }^{4-6}$ The sequential segmental approach advocated by those working in Europe 
added junctional morphology to these considerations. ${ }^{7-9,11}$ Others have now sought to combine the approaches. ${ }^{3,12}$ In this review, we discuss these various complementary methodologies in the context of heterotaxy. We then suggest concise definitions for the heterotaxy syndrome, and a variety of related terms. Part of the difficulty in describing patients with heterotaxy has been the fact that, when describing the atriums and ventricles, and their spatial relationships, the words "left" and "right" can be confusing. In the appendix, therefore, we suggest rules that can be used to provide consistency and accuracy when describing anatomical phenotypes with regards to the words right and left.

\section{Cardiac relations, and the way cardiac structures are joined together}

The segmental approach developed by Van Praagh and his colleagues ${ }^{13,14}$ documents the anatomy of the cardiac components, and the relations of the three major cardiac segments, namely, the atrial chambers, the ventricles, and the arterial trunks. Letters are coded in braces, also known as curly brackets " \{\} " to describe the segments as follows:

- the sidedness of the atrial chambers, or, in other words, the atrial "situs",

- the ventricular topology, in other words, the ventricular "loop", and

- the relationships of the arterial trunks in space

In this system, the atrial situs is coded with "S" for "situs solitus", otherwise known as normal arrangement; "I" for "situs inversus", or the mirror-imaged arrangement, " $A$ " for situs ambiguus, a term defined and discussed later in this manuscript, or " $\mathrm{X}$ " for unknown. Description of the ventricular loop is dependent on the topology and chirality, or handedness, of the ventricular mass. The system provides two choices, "D" for D-loop and "L" for L-loop, as defined below. Many centres also permit the designation of the chirality of the ventricular loop as "X", for those instances where the looping cannot be determined. The relationships of the arterial trunks is described with a code chosen from "S" for normally related great arteries, "I" for inverted, or mirror-imaged normally related great arteries, "D" for D-transposed or D-malposed great arteries, with the aorta to the right of the pulmonary trunk, "L" for L-transposed or L-malposed great arteries, with the aorta to the left of the pulmonary trunk, or " $A$ " for the aorta directly anterior to the pulmonary trunk. In this system, a normal heart is coded " $\{\mathrm{S}, \mathrm{D}, \mathrm{S}\}$ ". This coding system employing three letters does not specify the way the cardiac chambers within the segments are joined together, a feature that many describe as the type of "atrioventricular and ventriculoarterial connections". This feature, known in the school using the approach of Van Praagh as "atrioventricular and ventriculo-arterial alignments", is separately specified.

The system forming the basis of the European Paediatric Cardiac Code documents the anatomy of the cardiac components, as well as the junctions between them, using the sequential segmental approach. The atrial chambers, on the basis of the morphology of their appendages, are held to be usual or normally arranged, mirror-imaged, or to show isomerism of the morphologically right or left atrial appendages. The arrangements at the atrioventricular junctions take note of how the atrial and ventricular chambers are joined together, noting the possibilities for concordant, discordant, biventricular and mixed, double inlet, and absent connections. Separate consideration is given to the valves guarding the junctions, with the possibilities being two perforate valves, one single perforate valve with an absent atrioventricular connection, one perforate along with one imperforate valve, a common valve, or an absent valve with a so-called unguarded orifice. An imperforate valve is a structure formed by union of valvar leaflets so as to block completely the existing junction between adjacent structures, either an atrium and a ventricle, or a ventricle and an arterial trunk. Such an imperforate valve is different from a muscular wall of a chamber, since perforating the valve recreates the initial channel present between the adjacent structures. Either an atrioventricular valve or an arterial valve can be imperforate, and recognised as such by tomographic techniques or at autopsy. In addition, either or both atrioventricular valves may override or straddle the ventricular septum. The ventriculoarterial junctions are described in terms of the way the arterial trunks take origin from the ventricles, the infundibular morphologies, the morphology of the arterial valves, and the relationships of the arterial trunks in space. Specification of the arrangement of the atrial appendages, the atrioventricular connections, and the ventriculoarterial connections, does not specify nor imply the ventricular topology, or the relationships of the cardiac chambers or great arteries in space. These variables are separately specified.

\section{Bodily arrangement or "situs"}

The arrangement of the atrial chambers is determined on the basis of their sidedness within the body. The development of morphologically rightsided structures on one side of the body, and morphologically left-sided structures on the other side, is termed lateralization. Normal lateralization, 
the usual arrangement, is also known as "situs solitus". The mirror-imaged arrangement is also known as "situs inversus". The term "visceroatrial situs" is often used to refer to the situs of the viscera and atria when their situs is in agreement. The arrangement of the organs themselves, and the arrangement of the atrial chambers, is not always the same. ${ }^{5,11}$ Should such disharmony be encountered, the sidedness of the organs and atrial chambers must be separately specified.

When considering the arrangement of the organs, the school of nomenclature developed using the teachings of Van Praagh recognises three patterns. Situs solitus is the usual arrangement, and situs inversus is the mirror-imaged variant of solitus. The third pattern is situs ambiguus, which is defined as any situation where a combination of situs solitus and situs inversus occurs in the same individual.

In the normal atrial arrangement, or "atrial situs solitus", the morphologically right atrium is on the right, and the morphologically left atrium is on the left. In the mirror-imaged atrial arrangement, or "atrial situs inversus", the morphologically left atrium is on the right, and the morphologically right atrium is on the left. In terms of overall morphology, it is usually easy to differentiate the morphologically right atrium from the morphologically left atrium. This distinction can generally be made on the basis of the anatomy of the atrial appendages, the morphology of the atrial septum, and the drainage of the supradiaphragmatic portion of the inferior caval vein. Typically, the morphologically right atrial appendage is broad and blunt, whereas the morphologically left atrial appendage is narrow, pointed, and fingerlike. The morphologically right side of the atrial septum contains the rim of the oval fossa, or "limbus of the fossa ovalis", whereas the morphologically left side of the interatrial septum is made up of the flap valve of the oval fossa. When the organs themselves are lateralized, the supradiaphragmatic termination of the inferior caval vein provides an extremely reliable landmark for the morphologically right atrium. ${ }^{11}$ During echocardiographic examinations, the arrangement of the atrial chambers, also known as "atrial situs", is often inferred by documenting the location of the inferior caval vein within the abdomen, specifically by determining its relationship to the descending thoracic aorta in subcostal short and long axis views. In the normal atrial arrangement, or atrial "situs solitus", the aorta and inferior caval vein typically lie apart, on opposite sides of the spine, with the aorta on the left. This arrangement is mirrorimaged in atrial "situs inversus", with the aorta on the right and the inferior caval vein on the left.

The rules as stated above, however, are reliable only in the setting of usual or mirror-imaged arrangements. In the setting of heterotaxy, these relationships do not pertain. ${ }^{15}$ When there is isomerism of the right atrial appendages, as defined below, or "asplenia syndrome", the aorta and inferior caval vein are almost always on the same side of the spine, with the vein slightly anterior. In the setting of isomerism of the left atrial appendages, or "polysplenia syndrome", the inferior caval vein usually does not connect directly with the right atrium, its suprarenal course often being interrupted, with the blood returning to the heart through the azygos or hemiazygos venous tributaries, such that the aorta is midline and the azygos vein is located in a posterolateral position. ${ }^{15}$ In a crucial publication in $1980^{16}$ which appeared as a "Letter to the Editor" concerning a publication from the European school, ${ }^{17}$ Van Praagh and his colleagues established the "morphological method" as the optimal means of distinguishing between structures within the congenitally malformed heart. In essence, they stated that any variable structure within the heart should be identified on the basis of its own intrinsic morphology, and not on the basis of another feature that is itself variable. Applying this concept to malformed atrial chambers rules out the venoatrial connections, and also the relationships of the great vessels within the abdomen, as the most appropriate markers of atrial arrangement, since these features are frequently anomalous, particularly in the setting of heterotaxy. ${ }^{4,10}$

A feature of the atrial chambers does exist, however, that retains its value even in the setting of the malformed hearts seen in heterotaxy. The study of Uemura and colleagues, ${ }^{18}$ based on examination of hearts from more than 180 patients with heterotaxy, showed that the extent of the pectinate muscles relative to the vestibules of the right- and left-sided atrioventricular junctions distinguished between the morphologically right and left atrial appendages. In the normal morphologically right atrium, which of course is right-sided, the pectinate muscles extend all round the vestibule, and reach to the cardiac crux. In the normal left-sided morphologically left atrium, these pectinate muscles are confined within the tubelike left atrial appendage, and the smooth vestibule is confluent with the smooth-walled body of the left atrium. In patients with mirror-imaged arrangement, or "situs inversus", this morphological pattern is itself mirror-imaged.

"Isomerism" describes the situation in which morphologically right structures, or morphologically left structures, are found on both sides of the body in the same individual. The term is used in analogy to the situation in chemistry in which two compounds can have the same chemical structure, but be mirrorimages of each other. These compounds are called 

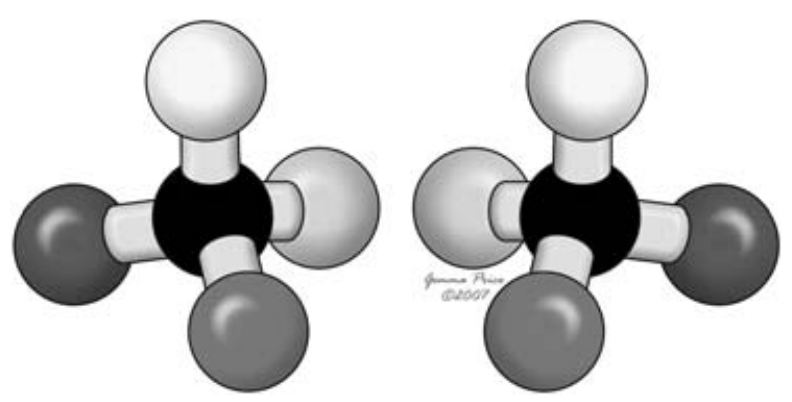

Figure 1.

The cartoon shows the situation of enantiomerism, or stereoisomerism. As is frequently found with the structure of chemical compounds, these two compounds are mirror-images of each other, although they have the same chemical structure.

enantiomers, or isomers, giving the arrangements of enantiomerism, or stereo-isomerism (Fig. 1). Such isomerism has long been known to exist in the lungs of patients with heterotaxy. Evidence of such isomerism also has been noted in the heart. Van Mierop and colleagues ${ }^{19,20}$ described the existence of right isomerism in the setting of asplenia syndrome, while Moller and colleagues ${ }^{21}$ pointed to the presence of bilateral left-sidedness in the setting of polysplenia syndrome. On the other hand, Van Praagh and Van Praagh believe that the concept of atrial isomerism is erroneous. ${ }^{22}$ The study of Uemura and colleagues, ${ }^{18}$ however, showed that the appendages when considered in isolation could truly be isomeric. In the hearts of all the patients with heterotaxy studied by Uemura and colleagues, ${ }^{18}$ it was noted that the pectinate muscles were arranged so as to produce the patterns of isomerism of either the right or left atrial appendages, hence their suggested stratification of heterotaxy, from the stance of the heart, into either "isomerism of the right atrial appendages" or "isomerism of the left atrial appendages" (Fig. 2). ${ }^{18}$ It is also pertinent to note that, subsequent to these morphological investigations proving the existence of isomerism of the atrial appendages when based on the feature of the extent of the pectinate muscles, molecular biologists have shown that it is also possible to produce isomerism within the atrial chambers of the heart in experimental animals. Thus, isomerism of the right atrial appendages is produced by knocking out either the Pitx2 or Cited 2 genes, ${ }^{23,24}$ whilst isomerism of the left appendages is produced by knocking out genes such as Sonic bedgehog. ${ }^{25}$

The presence of isomerism of the appendages within the atrial chambers, therefore, can be determined by the examination of the extent of the pectinate muscles relative to the atrioventricular junctions. The atrial chambers as a whole, however,

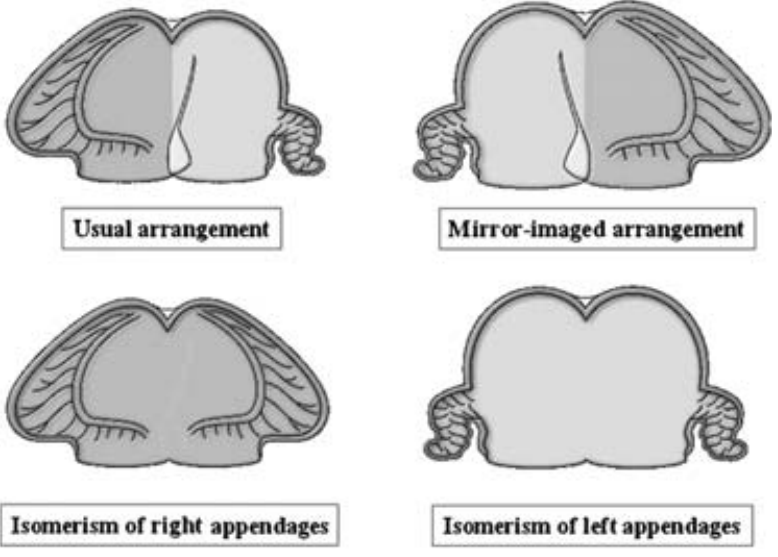

Figure 2 .

The cartoon shows how, when based on the extent of the pectinate muscles relative to the vestibules of the atrioventricular junctions, there are only four possible arrangements for the atrial appendages. The isomeric variants are the ones typically seen in patients with visceral heterotaxy.

are not isomeric. It is erroneous to describe "atrial isomerism", although this incorrect term is used widely and loosely. It is only the appendages that are isomeric in the setting of heterotaxy. Some cardiac morphologists, however, do not accept the concept of isomerism of the atrial appendages. They prefer to use the term atrial situs ambiguus to describe this subset of patients. To complicate matters, in the living patient, it may be difficult to document the extent of the pectinate muscles relative to the atrioventricular junctions. Because of this difficulty, note should be taken of the fact that, in heterotaxy, bronchopulmonary anatomy usually is consistent with the structure of the appendages, and can aid in the documentation of heterotaxy. Most patients with heterotaxy, of course, also have splenic abnormalities and the anatomy and structure of the spleen are often used to stratify patients with heterotaxy. Splenic anatomy, however, shows less correlation with the arrangement of the atrial appendages when compared to bronchopulmonary anatomy.

The morphology of the lungs, and the relation between the bronchial tree and the pulmonary arteries, therefore, are useful in determining "situs". The arrangement of the atrial appendages is highly consistent with bronchopulmonary morphology. ${ }^{11,26}$ In the majority of patients with heterotaxy, when attention is paid to the lungs and bronchial tree, the left-sided structures are seen to be the mirror-images of their right-sided counterparts (Fig. 3). A morphologically right lung typically has three lobes, and a morphologically left lung typically has two lobes. Furthermore, the right side tends to have an eparterial bronchus, whereas the 

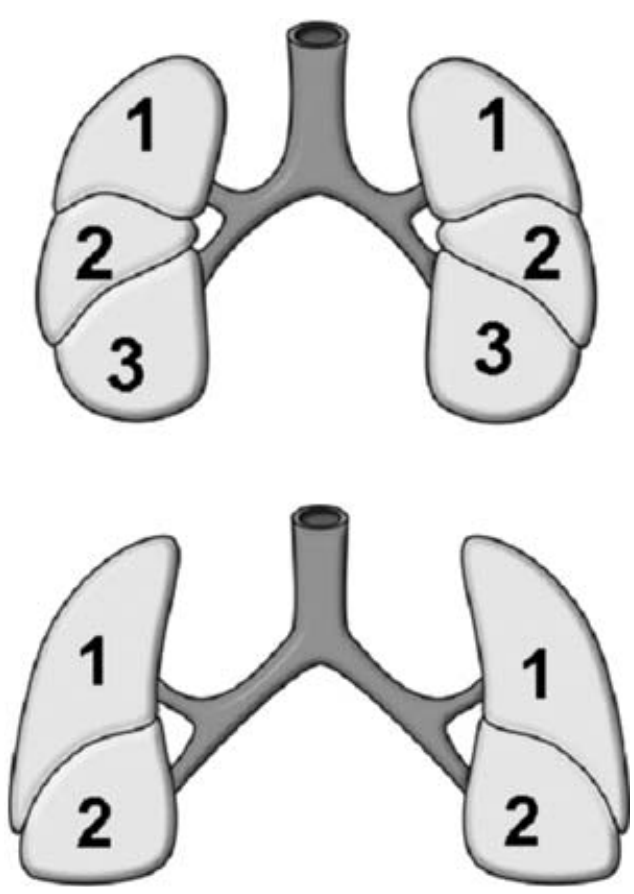

Figure 3.

The upper panel shows the typical arrangement of the lungs in the variant of visceral heterotaxy characterised by absence of the spleen. Each lung has 3 lobes, and is fed by a short bronchus, the usual arrangement for the morphologically right lung. The lower panel shows the typical arrangement seen in association with multiple spleens, with each lung having 2 lobes, and being supplied by a long bronchus, the arrangement usually seen in the morphologically left lung.

bronchus on the left side is typically hyparterial. An eparterial bronchus is one that branches superior to the first lobar division of the pulmonary artery, in contrast to a hyparterial bronchus that branches inferior to the first lobar division of the respective pulmonary artery. Tracheobronchial anatomy can be assessed from examination of the chest radiograph. ${ }^{11,27,28}$ Isomerism is consistent with a ratio of less than 1 to 1.6 between the lengths of the two main bronchuses, whereas lateralization has a ratio of more than 1 to 1.6. Examination of chest radiographs, therefore, may provide one of the simplest techniques for differentiating isomerism from lateralization in a living patient, albeit that this can now be shown with even greater detail using tomographic techniques. Bronchial tomography has been used to measure bronchial length, and comparison of measured bronchial length with the age of the patient has been used to determine the presence of right versus left bronchial isomerism. ${ }^{11,26,29}$ As already emphasised, nonetheless, whilst the correlation between bronchial morphology and the structure of the atrial appendages is highly consistent, it is not absolute. In patients with heterotaxy, the anatomy of the atrial appen- dages does not always correspond with the bronchial arrangement. ${ }^{30-32}$

Splenic anatomy is often used to stratify patients with heterotaxy. ${ }^{10,21}$ In most instances, right isomerism is associated with absence of the spleen, and left isomerism is associated with multiple spleens. This association, however, is weaker than the correlation between the arrangement of the atrial appendages and bronchial morphology. Moreover, absence of the spleen, or presence of multiple spleens, is not always easily documented. Multiple spleens are not always easy to differentiate from one spleen with several splenunculuses. ${ }^{10,21,33}$ A rudimentary spleen cannot easily be differentiated from one that is absent. ${ }^{34}$ Splenic anatomy can be difficult to determine both clinically and at autopsy. ${ }^{11}$

Although not all patients with multiple spleens have isomerism of the left atrial appendages, and not all patients with absence of the spleen have isomerism of the right atrial appendages, it has become customary for many paediatric cardiologists to stratify heterotaxy into the subsets of "asplenia syndrome" and "polysplenia syndrome". ${ }^{10,21}$ Because a syndrome includes a constellation of findings, each of which may not be present in all instances, and because the splenic arrangement does not always fit with the expected patterns of the remaining thoraco-abdominal organs, investigators may at times be comfortable with describing the presence of the spleen in patients known to have "asplenia syndrome". ${ }^{21}$ The challenge is magnified still further when we come to consider the heart, since one of the major features of the patients with heterotaxy is that they exhibit markedly abnormal venoatrial connections, as well as multiple and varied lesions within the heart. ${ }^{10,21}$ The start of cardiac analysis is the determination of the arrangement of the atrial chambers, and according to the morphological method, it is the structure of the appendages, the most constant atrial components, that is best fitted for determining this feature.

In any patient with a congenitally malformed heart, including those patients with heterotaxy, once the atrial arrangement is established, using the system with which the observer feels most comfortable, analysis proceeds with determining the morphology of the atrioventricular and ventriculoarterial junctions, including the way the various structures are or are not joined together, the ventricular topology, and the spatial arrangements of the arterial trunks, and then describing all the associated malformations, including the position of the heart and the orientation of its apex. In patients with heterotaxy, of course, it will also be necessary to describe the arrangements of the other systems of organs, since as we have discussed, these are not always as they are expected to be. The patient with 
isomerism of the morphologically right atrial appendages, for example, might well possess a spleen. The goal is to describe all such associations in precise fashion.

\section{Ventricular topology or "ventricular loop"}

The morphologically right ventricle typically possesses coarse trabeculations in its apical component, in contrast with the morphologically left ventricle which typically exhibits fine apical trabeculations. Ventricular topology, or "looping of the heart", describes the chirality, or handedness, of the ventricle mass. With right hand ventricular topology, or "D-loop", the right ventricle wraps around the left ventricle such that the palmar surface of the right hand can be placed on the septal surface of the right ventricle with the thumb in the inlet and the fingers in the outlet. With left hand ventricular topology, or "L-loop", it is the palmar surface of the left hand that fits on the right ventricular septum in this fashion. The concept of looping refers to the formation of the ventricular loop in the embryo. The loop normally rotates to form a D-loop.

In order to describe the fashion in which the atrial chambers are joined to the ventricular mass, this being the feature known variously as the atrioventricular connections or alignments, it is essential in any given patient first to take note of the atrial arrangement, and then to describe the specific ventricular topology. As will be discussed in the next section, patients with heterotaxy are similar to patients with those with lateralised arrangements because, in both groups of patients, the atrial chambers can be joined to the ventricles in biventricular or univentricular fashion.

\section{Atrioventricular junctions}

The term "atrioventricular connections", or "atrioventricular alignments", refers to the mechanism of union between the atrial and the ventricular myocardium. As already discussed, in any patient, in order to describe the fashion in which the atrial chambers are joined to the ventricular mass, it is essential first to take note of the atrial arrangement, and then to describe the specific ventricular topology. In this respect, the atrial chambers can be joined to the ventricles in biventricular or univentricular fashion. When joined in the univentricular fashion, the description in the setting of heterotaxy is exactly the same as for patients with usual or mirror-imaged arrangement, remembering of course that the univentricular arrangement is much more frequent when there are isomeric right as opposed to isomeric left atrial appendages. When

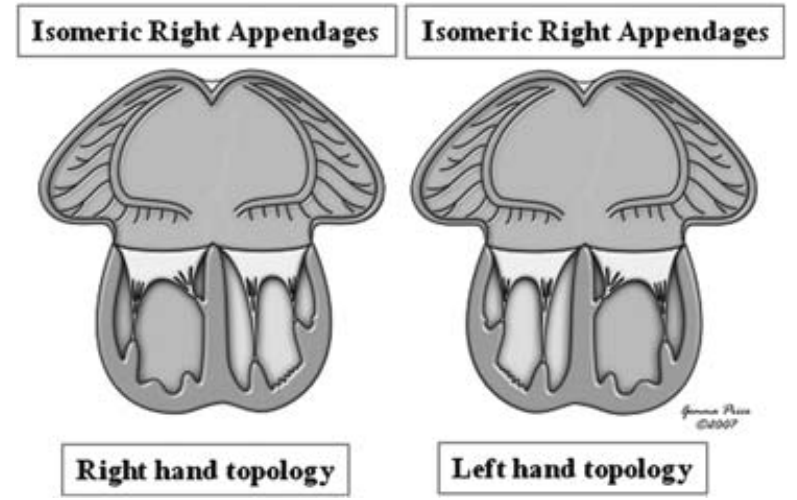

Figure 4 .

The cartoon shows how, in the setting of isomeric atrial appendages, be they of right (as shown here) or left morphology, and irrespective of the combination with right hand or left hand ventricular topology, and associated with biventricular atrioventricular connections, the union of the atrial and ventricular musculatures must be mixed in its pattern. In the European Paediatric Cardiac Code, this pattern is said to be "ambiguous", but "mixed" is a much better descriptor. Thus, the term "Mixed ('ambiguous') $A V$ connections (biventricular)," has been added to the version of the International Paediatric and Congenital Cardiac Code derived from the European Paediatric Cardiac Code.

the atrial chambers are joined to the ventricles in biventricular fashion, however, it should be noted that this pattern cannot exist in the setting of patients with lateralised atrial chambers. This anatomical phenotype is unique because, when the appendages are isomeric, and the atriums are joined to the ventricles in biventricular fashion, then irrespective of whether the isomeric appendages are morphologically left or right, and irrespective of the ventricular topology, half of the heart will be joined in concordant fashion, and the other half joined together in discordant fashion. In the setting of heterotaxy, therefore, biventricular atrioventricular connections or alignments are, of necessity, mixed (Fig. 4). Although many coding systems, including the European Paediatric Cardiac Code, have termed this pattern to be "ambiguous", the term "mixed" is probably a better descriptor, and has recently been incorporated into the International Paediatric and Congenital Cardiac Code.

As has already been discussed, analysis of the way the atrial myocardium is joined to the ventricular mass gives only half of the necessary information concerning the morphology of the atrioventricular junctions. It is also necessary to take account of the structure of the valve, or valves, guarding the junctions, in other words, the mode of the atrioventricular connection or alignment. A common atrioventricular junction, guarded by a common valve, is particularly frequent in the setting of isomeric right appendages, irrespective of whether there is a univentricular 
double inlet, or biventricular and mixed atrioventricular connections. Common junctions, guarded by common valves, are also relatively frequent in patients with isomerism of the left atrial appendages, but less common than in those with isomeric right atrial appendages. It is also possible, of course, to find separate atrioventricular junctions guarded by mitral and tricuspid valves in patients with isomeric right atrial appendages, but this is much less frequent.

\section{Ventriculoarterial junctions}

In patients with heterotaxy, after establishing the atrial arrangement, the ventricular topology, and the morphology of the atrioventricular junctions, the next step is description of the ventriculoarterial junctions. ${ }^{35,36}$ This analysis proceeds as for any other patient with a congenitally malformed heart, since all types of connection or alignment, arterial relationship, and infundibular and valvar morphology must be anticipated to exist. Certain patterns stand out as being associated with the two subsets of heterotaxy, but anything is possible. Discordant or double outlet ventriculoarterial connections or alignments, often times associated with severe pulmonary stenosis or atresia, are more frequent with isomerism of the right atrial appendages, or asplenia syndrome. Concordant ventriculoarterial connections, with subaortic obstruction and aortic coarctation, are more frequent in the setting of isomerism of the left atrial appendages, or polysplenia syndrome.

\section{Relations and connections or alignments}

The essence of the original segmental approach to diagnosis and nomenclature was analysis of the topological arrangement, or cardiac relations, of the atrial chambers, the ventricular mass, and the arterial trunks. ${ }^{37}$ When the European school ${ }^{38}$ sought to develop further this innovative methodology, they emphasized the importance also of describing the way the basic segments were united, or joined together, across their junctions, in other words, the cardiac connections or alignments. Very rarely, the segmental topological arrangements do not correspond with the way the chambers and arterial trunks are united across their junctions. ${ }^{39} \mathrm{It}$ is essential, therefore, that any system of nomenclature is able to distinguish between segmental topologies and junctional variations. ${ }^{3,39}$ This goal is important for all hearts, and especially those found in patients with heterotaxy. It may be accomplished either via the European approach, or the Bostonian approach developed by Van Praagh and his colleagues, as long as care is taken to specify both these features. 3,40

\section{The position of the heart and the orientation} of its apex

The position of the heart in the chest, and the orientation of the cardiac apex, must also be described separately, because these features can vary independently from each other, and have no definitive relationship to other cardiac relations and connections. The ventricular mass may be right-sided, left-sided, or midline. The cardiac apex may also be right-sided, left-sided, or midline. These features take on added importance when planning the route or pathway of an extracardiac Fontan connection.

The terms "dextrocardia", "levocardia", "mesocardia" "dextroversion", and "levoversion" have been used over the years in various fashions by various authors. ${ }^{41}$ "Dextrocardia" is most usually considered synonymous with a right-sided ventricular mass, whilst "dextroversion" is frequently defined as a configuration where the ventricular apex points to the right. In a patient with the usual atrial arrangement, or situs solitus, dextroversion, therefore, implies a turning to the right of the heart. In the same context, "levocardia" has at most times been used as synonymous with a left-sided ventricular mass, and "levoversion" is frequently defined as a configuration where the ventricular apex points to the left. "Mesocardia" is the term used to account for the ventricular mass occupying the midline. We should be cautious, however, about the use of such terms, the more so since others have used variations of this terminology, suggesting terms such as "dextrorotation", "mixed dextrocardia", or "pivotal dextrocardia" in attempts to compress all the information into a single term. These variations are not universally understood, and should be avoided.

The proper description of a heart in a patient with heterotaxy, irrespective of the system used, should include a description of the position of the heart in the chest and the orientation of the cardiac apex, mainly because these features can vary significantly in heterotaxy, and because they are not always in harmony. For example, one might encounter a patient with the cardiac mass may be located predominantly in the right chest, but with its apex pointing to the left, in other words, "dextrocardia with levoversion". The separate description of the location of the cardiac mass and the direction of the cardiac apex is not merely an academic exercise, since these features can profoundly impact planned surgical interventions.

\section{What about the venoatrial connections?}

A remarkable variety of venoatrial connections occur with heterotaxy. Indeed, it is the nature of these venoatrial connections that often dominates the clinical picture in patients with heterotaxy. 
In a small proportion of patients, all the systemic veins can return to one of the atrial chambers, and all the pulmonary veins to the other chamber. It is these settings in particular, which potentially produce quasi-concordant or quasi-discordant arrangements, ${ }^{42}$ and reduce the utility of the venoatrial connections as a surrogate for atrial "situs".

Take, for example, the situation as found in the patient with isomerism of the right atrial appendages, biventricular and mixed atrioventricular connections, left hand ventricular topology, but with all the pulmonary veins returning to the rightsided atrial chamber, possessing a morphologically right appendage, and all the systemic veins to the left-sided atrial chamber, also possessing a morphologically right appendage (Fig. 5). If defined on the basis of the venoatrial connections, this combination could well be interpreted as mirror-imaged atrial arrangement with concordant atrioventricular connections or alignments (Fig. 6). As shown in the two cartoons (Figs. 5 and 6), this interpretation would be incorrect, and would lead to inaccurate inferences regarding the location of the conduction tissues. This example demonstrates that, following the precepts of the "morphological method", ${ }^{18}$ the venoatrial connections cannot be used as the final arbiter of atrial arrangement.

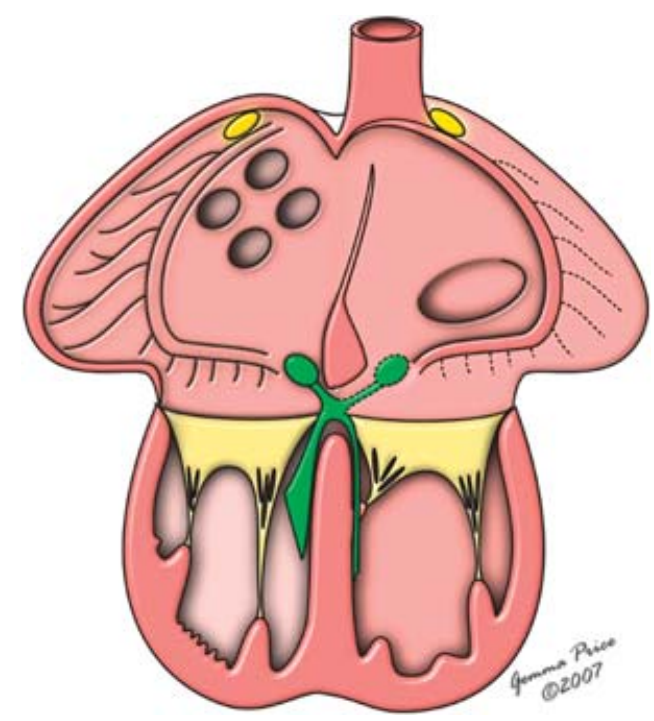

Figure 5.

The cartoon shows the situation in which there is isomerism of the right atrial appendages, biventricular and mixed atrioventricular connections, left hand ventricular topology, but with all the pulmonary veins returning to the right-sided atrial chamber, and all the systemic veins to the left-sided atrial chamber. Recognition of the isomeric arrangement permits the correct inference to be made concerning the bilateral nature of the sinus nodes (yellow ovals) and the likely presence of a sling of atrioventricular conduction tissue (green structures).
The venoatrial connections in patients with heterotaxy, therefore, must be described, rather than being used as an isolated indicator of atrial arrangement. It is then a fact that, even though all four pulmonary veins can drain to one of the atrial chambers in a minority of patients with isomeric right appendages, such connections are not anatomically normal. Indeed, even if the pulmonary veins do join the heart in patients with isomerism of the right atrial appendages, of necessity the connections themselves are anatomically abnormal since, in the absence of a morphologically left appendage, and other features of leftness, such as the presence of the coronary sinus in the atrioventricular junction, and possession of the flap valve of the septum, the atrial chamber to which they join cannot be a normal morphologically left atrium. Totally anomalous pulmonary venous connection, therefore, is an essential component of the heart in patients with isomeric right atrial appendages, as is absence of the coronary sinus. Patients with absence of the spleen may exhibit a coronary sinus, but they could not then have isomeric right atrial appendages. It is also the case that the coronary venous return is always abnormal when there are isomeric right atrial appendages. ${ }^{43}$ Abnormal coronary venous drainage can also be found in the setting of isomeric left

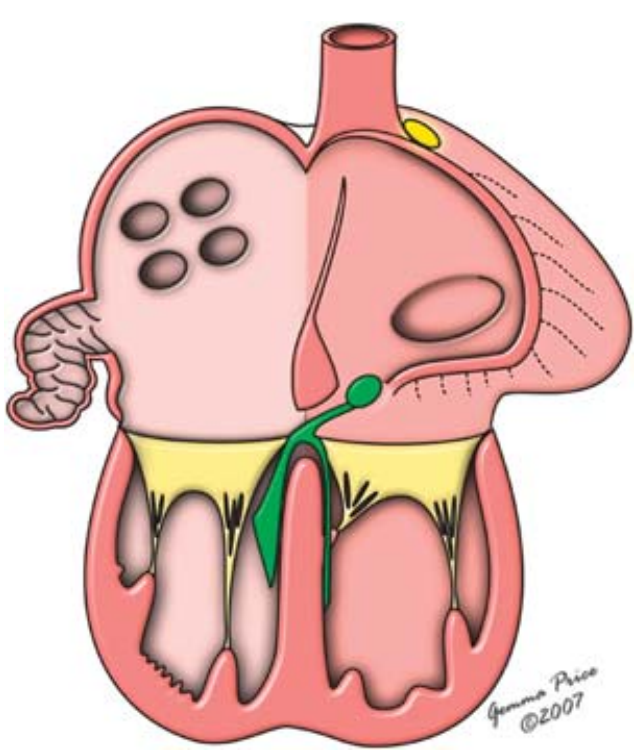

Figure 6.

Compare this situation with the arrangement shown in Figure 5. Had the venoatrial connections been used as the arbiters of atrial "situs", then the heart would have been presumed to show this arrangement, with left-sided sinus and atrioventricular nodes. This interpretation would obviously have been incorrect in the patient with isomeric right atrial appendages, showing that venoatrial connections cannot be used as the final arbiter of atrial arrangement. 
appendages should the coronary sinus be absent. Interruption of the inferior caval vein, with continuation through the azygos or hemiazygos venous system, is the most frequent anomalous venous connection found in those with isomerism of the left atrial appendages, or polysplenia syndrome, albeit that this is not a universal finding. Hepatic venous connections may also vary, especially when there are isomeric left appendages. Hepatic venous connections take on added significance when planning a Fontan operation, and especially completion of the Fontan operation after a prior superior cavopulmonary connection in the setting of an interrupted inferior caval vein with azygos continuation, in other words, a prior Kawashima operation. Indeed, apart from the absence of the coronary sinus, and the totally anomalous pulmonary venous connections, which are universally present in those with isomeric right atrial appendages, there are no other anomalous venous connections that serve to stratify perfectly the two subsets of patients with heterotaxy. Bilateral superior caval veins, for example, are frequent in both types, albeit that drainage via a coronary sinus can only be found in patients with isomerism of the left atrial appendages.

\section{Other associated malformations}

For patients with heterotaxy, the malformations within the heart are analysed and described as for any patient with suspected congenital cardiac disease. Again, there is no specific lesion that serves to stratify the two subsets of heterotaxy. Each individual associated cardiac lesion must be described.

Careful attention also should be paid to the arrangement of the extracardiac structures, since although an essential feature of heterotaxy, there is often discrepancy between the morphology of the atrial appendages and the position of the thoracoabdominal organs. ${ }^{44}$ The arrangement of the lungs and spleen must be described separately. Intestinal malrotation is another frequently associated lesion that must be considered. Some advocate that all patients with heterotaxy, especially those with isomerism of the right atrial appendages or asplenia syndrome, should have a barium study to evaluate for intestinal malrotation, given the associated potential morbidity. The cardiac anatomy and associated cardiac malformations, as well as the relationship and arrangement of the remaining thoraco-abdominal organs, must be described separately.

\section{Clinical limitations and challenges}

Although the above described step-wise approach to the analysis of the cardiac morphology can be used to make the precise anatomic diagnosis and classification in pathological specimens, it cannot always produce diagnostic certainty in the clinical arena. Ascertaining the precise anatomic diagnosis and classification can be very challenging in the living patient with heterotaxy. The step-wise approach to the analysis of the cardiac morphology will increase the likelihood of ordered thought and analysis. It must be recognized, nonetheless, that there are patients in whom the combination of inadequate images, conflicting observations, and morphologic uncertainty, preclude diagnostic certainty. Clearly, much of this uncertainty can be resolved by examination of the explanted heart. There are patients, nevertheless, in whom the clinicians cannot reach agreement as to the ventricular loop, the morphology of the atrioventricular valve, and particularly the anatomy of the atrial appendages and their pectinate muscles, despite use of multiple diagnostic modalities, such as echocardiography, magnetic resonance imaging, cardiac catheterization, and direct surgical observation. For example, the surgeon will rarely have the opportunity to have both atrial appendages laid completely open to verify the extent and direction of the pectinate muscles, and in some instances may be unable to state with absolute certainty whether one is dealing with isomerism of the atrial appendages. Diagnostic uncertainty is a clinical reality, and must be acknowledged when present to avoid improperly placing a patient into a given diagnostic category.

\section{Definitions}

Based on the above discussion, and a thorough review of the literature, we offer the following definitions.

\section{Heterotaxy and heterotaxy syndrome}

The word heterotaxy is derived from the Greek: heteros- meaning other than, and taxis- meaning arrangement. Heterotaxy, therefore, literally means a pattern of anatomical organization of the thoracic and the abdominal organs which is not the expected usual or normal arrangement, also known as "situs solitus". In the strictest sense, patients with the mirror-imaged arrangement, also known as "situs inversus" should be included in the group of patients with heterotaxy or heterotaxy syndrome. It has become traditional, nonetheless, to use heterotaxy as a term to describe patients who have neither the normal nor the mirror-imaged arrangements. ${ }^{45}$ As used in the field of the congenitally malformed heart, the term "heterotaxy" has become 
conventional for description of those patients with jumbled-up organs, almost always with problems in formation of the spleen.

We reviewed the literature and found 50 published definitions of heterotaxy, many of them including the phrase "visceral heterotaxy". In many respects, however, "visceral heterotaxy" is tautologous, and "visceral" can be discarded without changing the basic understanding of "heterotaxy". Crucial features of heterotaxy are captured in the following two definitions:

- Heterotaxy is a class of congenital disorders resulting from failure to establish normal left right asymmetry during embryonic development. The resulting defects are characterized by segmental discordances along the left-right axis. ${ }^{46}$

- Heterotaxy is defined as the abnormal arrangement of organs or viscera across the left-right axis differing from "complete situs solitus" and "complete situs inversus". 47

In the normal body, parietal structures such as hands and feet are mirror images of each other, while the internal organs are arranged in different fashions on the right and left sides, and are not mirror images of each other. As previously discussed, the development of morphologically right-sided structures on one side of the body and morphologically left-sided structures on the other side is termed "lateralization". While parietal structures are normally mirror images of each other, internal organs are normally lateralized. Hence, if asymmetry of the thoracic and abdominal organs is the usual or normal situation, the syndrome of visceral heterotaxy includes patients with an unusual degree of symmetry of some of the thoracic and abdominal organs, and the atrial appendages within the heart. This broad term includes patients with a wide variety of very complex cardiac lesions. A "syndrome", meanwhile, is a group of signs and symptoms that occur together, and characterize a particular abnormality. ${ }^{2}$ A syndrome includes a constellation of findings, each of which may not be present in all instances. On this basis, therefore, the Nomenclature Working Group offers the following definition for the term "heterotaxy":

\footnotetext{
"Heterotaxy is synonymous with 'visceral heterotaxy' and 'heterotaxy syndrome'. Heterotaxy is defined as an abnormality where the internal thoraco-abdominal organs demonstrate abnormal arrangement across the left-right axis of the body. By convention, heterotaxy does not include patients with either the expected usual or normal arrangement of the internal organs along the left-right axis, also known as 'situs solitus', nor patients with complete mirror-imaged arrangement of the internal organs along the left-right axis also known as 'situs inversus'."
}

Patients with heterotaxy can be stratified into the subsets of asplenia syndrome and polysplenia syndrome, or the subsets of heterotaxy with isomerism of the right atrial appendages and heterotaxy with isomerism of the left atrial appendages.

\section{Isomerism}

The word isomerism is also derived from the Greek: isos- meaning equal and meros- meaning part. Isomerism refers to structures that are themselves mirror-imaged. The term has been used extensively in the field of chemistry, where it has a very precise definition. In chemistry, isomers are defined as different compounds that have the same molecular formula. ${ }^{48}$ The term "structural isomers" is synonymous with the term "constitutional isomers", and is defined as isomers that differ because their atoms are joined in a different order. ${ }^{48}$ On the other hand, stereoisomers are not structural isomers, because they have their constituent atoms attached in the same order. Stereoisomers are isomers that differ only in the arrangement of their atoms in space (Fig. 1). Stereoisomers, therefore, are defined as different compounds that have the same molecular formula, and have their constituent atoms attached in the same order, but differ from each other only in the arrangement of their atoms in space. ${ }^{48}$ Such stereoisomers are enantiomers when their molecules are mirror reflections of each other. ${ }^{48}$ Diastereomers are stereoisomers whose molecules are not mirror reflections of each other. ${ }^{48}$

When used in the arena of the congenitally malformed heart, the term "isomerism" has become the conventional description for the situation in which morphologically right structures or morphologically left structures are found on both sides of the body in the same individual. When used in this fashion, the term is analogous to the chemical term enantiomers. In the normal body, parietal structures, such as hands and feet, are good examples of enantiomerism, or bodily isomerism. In heterotaxy, some of the paired internal organs, and the atrial appendages within the heart, may also display such enantiomerism or bodily isomerism.

On this basis, therefore, the Nomenclature Working Group offers the following definition for the term "isomerism":

"Isomerism in the context of the congenitally malformed heart is defined as a situation where some paired structures on opposite sides of the left-right axis of the body are, in morphologic terms, symmetrical mirror images of each other." 


\section{Left isomerism}

"Left isomerism in the context of the congenitally malformed heart is defined as a subset of heterotaxy where some paired structures on opposite sides of the left-right axis of the body are symmetrical mirror images of each other, and have the morphology of the normal left-sided structures."

Those having isomeric left atrial appendages frequently have bilaterally bi-lobed lungs, each with a long bronchus, and multiple spleens. In many cases of isomeric left appendages, patients have pulmonary veins connecting to both atrial chambers, as if both atriums were morphologically left atriums. The symmetry, however, is far from complete. Whilst all patients with isomeric left appendages, for example, have at least one superior caval vein, no patient has yet been described, to the best of our knowledge, with two superior caval veins, each draining to a coronary sinus, as might be expected if the patient possessed two complete left atrial chambers. Examples exist where the sidedness of the atrial appendages is not concordant with the sidedness of the lungs or the arrangement of the spleen. It is necessary in such disharmonious situations to describe separately the arrangement of the atrial appendages, the lungs, and the spleen.

\section{Right isomerism}

"Right isomerism in the context of the congenitally malformed heart is defined as a subset of heterotaxy where some paired structures on opposite sides of the left-right axis of the body are symmetrical mirror images of each other, and have the morphology of the normal right-sided structures."

Those having isomeric right atrial appendages frequently have bilaterally tri-lobed lungs, each with a short bronchus, and absence of the spleen. Once again, however, examples exist where the sidedness of the atrial appendages is not concordant with the sidedness of the lungs or spleen. In these situations, it will be necessary to describe separately the arrangement of the atrial appendages, the lungs, and the spleen.

\section{Atrial isomerism versus isomerism of the atrial appendages}

Atrial isomerism does not exist, and therefore cannot be defined. It is only the atrial appendages that are isomeric in the setting of heterotaxy. One way to impose order upon the diverse group of cardiac lesions within the heterotaxy syndrome is to stratify them according to the morphology of the atrial appendages. ${ }^{18}$ As already explained, in the setting of heterotaxy, the atrial appendages on the two sides of the heart have comparable rather than their usual disparate morphology. Isomerism of the right or left atrial appendages exists when both atrial chambers have appendages with the same morphology. On this basis, therefore, the Nomenclature Working Group offers the following definitions for the "isomerism of the atrial appendages":

\section{Isomerism of the left atrial appendages}

"Isomerism of the left atrial appendages is a subset of heterotaxy where the atrial appendages on both sides of the body have the appearance of the morphologically left atrial appendage."

\section{Isomerism of the right atrial appendages}

"Isomerism of the right atrial appendages is a subset of heterotaxy where the atrial appendages on both sides of the body have the appearance of the morphologically right atrial appendage."

\section{Situs ambiguus}

"Situs ambiguus is defined as an abnormality in which there are components of situs solitus and situs inversus in the same person. Situs ambiguus, therefore, can be considered to be present when the thoracic and abdominal organs are positioned in such a way with respect to each other as to be not clearly lateralised and thus have neither the usual, or normal, nor the mirrorimaged arrangements."

All patients with heterotaxy also may be considered to have "situs ambiguus", and all patients with "situs ambiguus" also have heterotaxy syndrome. Of course, it is obvious by now that individual description of any system of organs produces specificity, as far as the morphology permits such specificity. Although isomerism of the atrial appendages meets the above definition of "situs ambiguus", the presence of bilateral right atrial appendages or bilateral left atrial appendages, when present, can often be described specifically. The term "visceroatrial situs ambiguus" has more application, however, because of the frequent discordance between the arrangement of the atrial appendages and the other organs. Because of the numerous examples of inconsistency between the arrangement of the atrial appendages and the other organs, some prefer to utilize the term "situs ambiguus" for everything that is not "situs solitus" or "situs inversus". If the definitions currently suggested are found acceptable, this use of the term situs ambiguus will be the same as stating that such patients have heterotaxy. Within this group of 
patients classified as visceroatrial situs ambiguus, nevertheless, specificity can be provided in the individual patient by accounting separately for the sidedness of the atrial appendages, the lungs and the abdominal organs.

\section{Asplenia and asplenia syndrome}

Asplenia literally means absent spleen. Asplenia can be isolated.

"Asplenia syndrome" can be defined as a subset of heterotaxy with components of bilateral right-sidedness, usually associated with absence of the spleen.

In the context of heterotaxy, asplenia is usually, but not always, accompanied by a variety of cardiac malformations that may include isomeric right atrial appendages, common atrioventricular junction, abnormal ventriculoarterial connections or alignments, also termed "conotruncal anomalies", and a high incidence of pulmonary atresia or pulmonary stenosis, and totally anomalous pulmonary venous connection. Virtually all patients with asplenia syndrome have a common atrioventricular junction, as do almost two-thirds of those with polysplenia syndrome. Asplenia syndrome is not typically associated with interruption of the inferior caval vein or drainage of the superior caval vein to the coronary sinus. Patients having asplenia syndrome as defined above, however, may possess a single spleen of normal size, or even multiple spleens.

While patients with absence of the spleen may rarely be found with drainage of the superior caval vein to the coronary sinus, this association cannot be found in the setting of isomeric right atrial appendages, since the coronary sinus is universally absent when both appendages are of right morphology. Thus, although the spleen is expected to be absent in the setting of isomeric right atrial appendages, this association does not always coexist.

\section{Polysplenia and polysplenia syndrome}

Polysplenia literally means multiple spleens. In some instances, patients with usual arrangement of the atrial appendages can have multiple spleens, as can those with isomeric right atrial appendages.

"Polysplenia syndrome" can be defined as a subset of heterotaxy with components of bilateral left-sidedness, usually associated with multiple spleens.

In the context of heterotaxy, polysplenia is usually, but not always, accompanied by significant cardiac disease, with or without a common atrioventricular junction, and with or without malformations of the outflow tracts, also known as "conotruncal anomalies". Those with polysplenia syndrome typically have interruption of the inferior caval vein, and often have pulmonary veins from the two sides of the body draining in symmetrical fashion to atriums each having a morphologically left appendage. In this setting, the venoatrial connections themselves will be bilateral, but anatomically normal. Patients with multiple spleens typically have isomeric left atrial appendages, but also can have usually arranged or mirror-imaged atrial appendages, or rarely isomeric right atrial appendages.

Most of the associations with asplenia syndrome and polysplenia syndrome are less than perfect, with overlap between the two. ${ }^{49}$ One of the clinically important implications of heterotaxy syndrome is that splenic abnormalities are common. Investigation of any patient with the cardiac findings associated with heterotaxy, therefore, should include analysis of splenic morphology. The less than perfect association between the state of the spleen and the form of heart disease implies that splenic morphology should be investigated in all forms of heterotaxy, regardless of the type of cardiac disease. The splenic morphology should not be used to stratify the form of disease within the heart, and the form of cardiac disease should not be used to stratify the state of the spleen.

\section{Ivemark syndrome}

Ivemark syndrome $e^{50}$ is a term that, historically, is synonymous with asplenia syndrome. Other synonyms have been used for heterotaxy syndrome with asplenia including asplenia syndrome, bilateral right-sidedness sequence, and splenic agenesis syndrome. $^{51}$ As discussed above, heterotaxy syndrome with asplenia is characterized by the absence of a spleen, malformations of the cardiovascular system, and abnormal arrangement of the thoracoabdominal organs. ${ }^{52}$

\section{Nomenclature crossmap for heterotaxy and its related diagnoses}

In Table 1, we show the terminology for diagnoses related to heterotaxy from the version of the International Pediatric and Congenital Cardiac Code derived from the list of the Society of Thoracic Surgeons and the European Association of CardioThoracic Surgery. In Table 2, we show the equivalent and crossmapped terms from the version derived from the list of the Association for European Paediatric Cardiology. 
Table 1. The European Association for Cardiothoracic Surgery - Society of Thoracic Surgeons derived version of the International Paediatric and Congenital Cardiac Code for diagnoses related to heterotaxy.

Normal atrial arrangement (situs), AV \& VA connections

Normal heart

Normal pulmonary venous connections

Normal systemic venous connections

Atrial appendage isomerism

Atrial appendage isomerism, Left

Atrial appendage isomerism, Right

Atrial situs (arrangement) abnormal

Atrial situs (arrangement) ambiguus

Atrial situs (arrangement) unknown

Atrial situs inversus (Mirror image atrial arrangement)

Atrial situs solitus (Usual atrial arrangement)

$\mathrm{AV}$ connection $(\mathrm{s})=$ Absent (single inlet ventricle)

AV connection(s) $=$ Absent left sided AV connection

$\mathrm{AV}$ connection $(\mathrm{s})=$ Absent left sided AV connection - univentricular

AV connection(s) = Absent left sided AV connection with straddling valve - uniatrial biventricular

AV connection(s) = Absent left sided AV connection, Right-sided atrium to both ventricles

$\mathrm{AV}$ connection(s) $=$ Absent left sided AV connection, Right-sided atrium to LV

AV connection(s) $=$ Absent left sided AV connection, Right-sided atrium to RV

$\mathrm{AV}$ connection(s) $=$ Absent left sided AV connection, Right-sided atrium to ventricle of indeterminate morphology

AV connection(s) $=$ Absent right sided AV connection

$\mathrm{AV}$ connection(s) $=$ Absent right sided AV connection - univentricular

AV connection(s) = Absent right sided AV connection with straddling valve - uniatrial biventricular

AV connection(s) = Absent right sided AV connection, Left-sided atrium to both ventricles

$\mathrm{AV}$ connection(s) $=$ Absent right sided AV connection, Left-sided atrium to LV

$\mathrm{AV}$ connection(s) $=$ Absent right sided AV connection, Left-sided atrium to RV

$\mathrm{AV}$ connection(s) = Absent right sided AV connection, Left-sided atrium to ventricle of indeterminate morphology

AV connection(s) $=$ Discordant AV connections (biventricular)

AV connection $(s)=$ Double inlet AV connection (univentricular)

AV connection(s) $=$ Double inlet AV connection (univentricular), Double inlet LV

$\mathrm{AV}$ connection(s) = Double inlet AV connection (univentricular), Double inlet RV

$\mathrm{AV}$ connection(s) = Double inlet AV connection (univentricular), Double inlet to solitary ventricle of indeterminate morphology

AV connection(s) = Double inlet AV connection (univentricular): Mode of connection $=$ Common AV orifice in double inlet ventricle

$\mathrm{AV}$ connection $(\mathrm{s})=$ Double inlet $\mathrm{AV}$ connection (univentricular): Mode of connection $=$ Two AV valves in

double inlet ventricle

AV connection(s) $=$ Double inlet AV connection (univentricular): Mode of connection $=$ Unknown

AV connection(s) = Mixed ("Ambiguous") AV connections (biventricular)

AV connection(s) = Normal atrioventricular connections (Concordant atrioventricular connections in

biventricular heart)

AV connection(s) = Unknown atrioventricular connections

AV valve, Imperforate

AV valve, Imperforate, Left sided AV Valve

AV valve, Imperforate, Mitral valve

AV valve, Imperforate, Right sided AV Valve

AV valve, Imperforate, Tricuspid valve

AV valve overriding

AV valve overriding, Left sided AV Valve

AV valve overriding, Mitral valve

AV valve overriding, Right sided AV Valve

AV valve overriding, Tricuspid valve

AV valve overriding-modifier for degree of override

$A V$ valve overriding-modifier for degree of override, Override of AV valve $<50 \%$

AV valve overriding-modifier for degree of override, Override of AV valve $>90 \%$

AV valve overriding-modifier for degree of override, Override of AV valve 50-90\%

AV valve straddling

AV valve straddling, Left sided AV Valve

AV valve straddling, Mitral valve

AV valve straddling, Right sided AV Valve

AV valve straddling, Tricuspid valve
01.03 .10

01.01 .00

04.00 .03

04.00 .00

01.03 .08

01.03 .03

01.03 .02

01.03 .06

01.03 .04

01.03 .07

01.03 .01

01.03 .00

01.04 .21

01.04 .20

01.04 .13

01.04 .16

$01.04 .20,01.04 .18$

$01.04 .20,01.04 .10$

$01.04 .20,01.04 .09$

$01.04 .20,01.04 .11$

01.04 .19

01.04 .12

01.04 .15

$01.04 .19,01.04 .17$

$1.04 .19,01.04 .06$

$01.04 .19,01.04 .07$

$01.04 .19,01.04 .08$

01.04.01

01.01 .14

01.04 .04

01.04 .03

01.04 .05

01.06 .02

01.06 .01

01.04 .23

01.04 .02

01.04 .00

01.04 .22

06.00.07

06.00 .13

06.02 .02

06.00 .12

06.01 .02

06.00 .01

06.00 .03

06.02 .05

06.00 .02

06.01 .05

Title

Q1.27.74

Q1.27.76

Q1.27.75

06.00 .04

06.00 .06

06.02 .09

06.00 .05

06.01 .09 
VA connection $=$ Concordant VA connections with parallel great arteries (anatomically corrected malposition)

VA connection $=$ Normal ventriculoarterial connections (Concordant ventriculoarterial connections in

biventricular heart)

VA connection $=$ Single outlet VA connection

VA connection $=$ Single outlet VA connection, Common arterial trunk

VA connection $=$ Single outlet VA connection, Single outlet VA connection via aorta (pulmonary atresia)

09.01 .01

VA connection $=$ Single outlet VA connection, Single outlet VA connection via pulmonary trunk

01.05 .32

(aortic atresia)

VA connection $=$ Single outlet VA connection, Solitary arterial trunk (absent intrapericardial

01.05 .33

pulmonary arteries)

VA connection $=$ Single outlet VA connection-modifier for ventricular commitment of single

09.07 .26

VA outlet artery

VA connection $=$ Single outlet VA connection-modifier for ventricular commitment of single VA outlet artery,

Single ventricular outlet above both ventricles

VA connection $=$ Single outlet VA connection-modifier for ventricular commitment of single VA outlet artery,

Single ventricular outlet above LV

VA connection $=$ Single outlet VA connection-modifier for ventricular commitment of single VA outlet artery,

Single ventricular outlet above RV

VA connection $=$ Single outlet VA connection-modifier for ventricular commitment of single VA outlet artery,

Single ventricular outlet above ventricle of indeterminate morphology

VA connection $=$ Single outlet VA connection-modifier for ventricular commitment of single VA outlet artery, Unknown

VA connection $=$ Unknown ventriculoarterial connections

VA connection $=$ Discordant VA connections $(T G A)$

VA connection $=$ Double outlet VA connections

VA connection $=$ Double outlet VA connections, Double outlet from ventricle of indeterminate morphology

VA connection $=$ Double outlet VA connections, Double outlet LV

VA connection $=$ Double outlet VA connections, Double outlet RV

VA connection $=$ Double outlet VA connections, Double outlet RV, "TGA type" (Subpulmonary VSD)

("Transposition type")

VA connection $=$ Double outlet VA connections, Double outlet RV, "TOF type" ("Fallot type")

VA connection $=$ Double outlet VA connections, Double outlet RV, "VSD type"

VA connection $=$ Double outlet VA connections, Double outlet RV, Remote VSD (Uncommitted VSD)

VA connection $=$ Double outlet VA connections, Double outlet RV, With doubly committed subarterial VSD

VA connection $=$ Double outlet VA connections, Double outlet RV, With intact ventricular septum (IVS)

VA connection $=$ Double outlet VA connections-modifier for VSD in double outlet VA connection

VA connection $=$ Double outlet VA connections-modifier for VSD in double outlet VA connection,

Doubly committed

VA connection $=$ Double outlet VA connections-modifier for VSD in double outlet VA connection, Non-committed

VA connection $=$ Double outlet VA connections-modifier for VSD in double outlet VA connection, Subaortic

VA connection $=$ Double outlet VA connections-modifier for VSD in double outlet VA connection,

Subpulmonary

VA valve, Imperforate

VA valve, Imperforate, Aortic valve

VA valve, Imperforate, Left sided VA Valve

VA valve, Imperforate, Pulmonary valve

VA valve, Imperforate, Right sided VA Valve

Title

01.05 .41

01.05 .43

01.05 .42

01.05 .44

01.05 .46

01.05 .17

01.05 .01

01.01 .50

01.05 .04

01.05 .03

01.01 .04

01.01 .18

01.01 .17

01.01 .40

01.01 .19

01.01 .23

01.01 .24

07.13 .01

07.13 .02

07.13 .03

07.13 .04

07.13 .05

09.04 .22

09.15 .04

09.04 .25

09.05 .12

09.04 .26

09.04 .21

09.15 .47

VA valve overriding, Aortic valve

VA valve overriding, Left sided VA Valve

09.04 .24

VA valve overriding, Pulmonary valve

09.05 .52

09.04 .23

Title

VA valve overriding-modifier for degree of override

VA valve overriding-modifier for degree of override, Override of VA valve $<50 \%$

VA valve overriding-modifier for degree of override, Override of VA valve $>90 \%$

Q1.27.98

Q1.28.00

$\begin{array}{ll}\text { VA valve overriding-modifier for degree of override, Override of VA valve 50-90\% } & \text { Q1.27.99 }\end{array}$

Aortic arch, Abnormal course of aortic arch

Aortic arch, Abnormal course of aortic arch and descending aorta

09.28 .23

09.28 .19

Aortic arch, Aortic arch centrally descending

09.28 .05

Aortic arch, Left aortic arch

09.28 .22

Aortic arch, Left aortic arch branching pattern

09.30 .25 
Aortic arch, Left aortic arch, Left aortic arch and left descending aorta

Aortic arch, Left aortic arch, Left aortic arch and right descending aorta

Aortic arch, Left aortic arch, Left aortic arch centrally descending

Aortic arch, Right aortic arch

Aortic arch, Right aortic arch (mirror image) branching pattern

Aortic arch, Right aortic arch, Right arch and left descending aorta

Aortic arch, Right aortic arch, Right arch and right descending aorta

Aortic arch, Right aortic arch, Right arch centrally descending

Segmental nomenclature, Letter 1 (Situs) $=\mathrm{A}=$ Ambiguus

Segmental nomenclature, Letter 1 (Situs) $=\mathrm{I}=$ Inversus

Segmental nomenclature, Letter 1 (Situs) $=\mathrm{S}=$ Solitus

Segmental nomenclature, Letter 1 (Situs) $=\mathrm{X}=$ Unknown or not possible to determine

Segmental nomenclature, Letter 2 (Loop) $=\mathrm{D}=\mathrm{D}$-Loop (Right hand pattern ventricular topology)

Segmental nomenclature, Letter 2 (Loop) $=\mathrm{L}=\mathrm{L}$-Loop (Left hand pattern ventricular topology)

Segmental nomenclature, Letter 2 (Loop) $=\mathrm{X}=$ Unknown or not possible to determine

Segmental nomenclature, Letter 3 (Relationship of great arteries in space) $=\mathrm{A}=$ Aorta directly anterior to pulmonary artery

Segmental nomenclature, Letter 3 (Relationship of great arteries in space) $=\mathrm{D}=$ Aorta to the right of the pulmonary artery

Segmental nomenclature, Letter 3 (Relationship of great arteries in space) $=\mathrm{L}=$ Aorta to the left of the pulmonary artery

Segmental nomenclature, Letter 3 (Relationship of great arteries in space) $=\mathrm{N}=$ Normal (Solitus)

Segmental nomenclature, Letter 3 (Relationship of great arteries in space) $=\mathrm{S}=$ Solitus (Normal)

Segmental nomenclature, Letter 3 (Relationship of great arteries in space) $=\mathrm{X}=$ Unknown or not possible to determine

$\mathrm{AV}$ and/or VA connections abnormal

Crisscross heart (twisted AV connections)

Crisscross heart (twisted AV connections), With leftward rotation

Crisscross heart (twisted AV connections), With rightward rotation

Cardiac apex anterior to the cardiac base

Cardiac apex posterior to the cardiac base

Cardiac apex bifid

Cardiac apex midline

Cardiac apex midline and anterior

Cardiac apex midline and posterior

Cardiac apex to the left

Cardiac apex to the right

Dextrocardia (right sided ventricular mass)

Dextrorotation (heart rotated to the right)

Dextroversion (ventricular apex points to the right)

Levocardia (left sided ventricular mass)

Levorotation (heart rotated to the left)

Levoversion (ventricular apex points to the left)

Mesocardia (central/midline ventricular mass)

Position and orientation of the heart in the thorax is abnormal

Position and orientation of the heart in the thorax is normal

Relationship of aortic orifice with respect to pulmonary orifice, Abnormal relationship of aortic orifice to pulmonary orifice

Relationship of aortic orifice with respect to pulmonary orifice, Aortic orifice anterior with respect to pulmonary orifice

Relationship of aortic orifice with respect to pulmonary orifice, Aortic orifice left and anterior with respect with respect to pulmonary orifice

Relationship of aortic orifice with respect to pulmonary orifice, Aortic orifice left and posterior with respect to pulmonary orifice

Relationship of aortic orifice with respect to pulmonary orifice, Aortic orifice left and side-by-side with respect to pulmonary orifice

Relationship of aortic orifice with respect to pulmonary orifice, Aortic orifice posterior with respect to pulmonary orifice

Relationship of aortic orifice with respect to pulmonary orifice, Aortic orifice right and anterior with respect to pulmonary orifice

Relationship of aortic orifice with respect to pulmonary orifice, Aortic orifice right and posterior with respect to pulmonary orifice (normal)

Relationship of aortic orifice with respect to pulmonary orifice, Aortic orifice right and side-by-side with respect to pulmonary orifice
09.28 .01

09.28 .02

$09.28 .22,09.28 .05$

09.28 .15

09.30 .26

09.28 .04

09.28 .03

$09.28 .15,09.28 .05$

01.03 .04

01.03 .01

01.03 .00

01.03 .07

02.03 .01

02.03 .02

02.03 .06

02.06 .03

02.06.02

02.06.04

02.06 .00

02.06 .00

02.06 .11

01.03 .09

02.03 .03

02.03 .11

02.03 .12

02.02 .20

02.02 .21

02.02 .03

02.02 .04

02.02 .18

02.02 .19

02.02 .00

02.02 .01

02.01 .02

02.01 .05

02.02 .01

02.01 .03

02.01 .06

02.02 .00

02.01 .04

02.01 .09

02.01 .00

02.06 .09

02.06 .03

02.06 .04

02.06 .06

02.06 .05

02.06.07

02.06 .02

02.06 .00

02.06.01 
Relationship of aortic orifice with respect to pulmonary orifice, Aortic sinuses malaligned with respect to pulmonary sinuses

Relationship of aortic orifice with respect to pulmonary orifice, Unknown

Mitral valve - aortic valve continuity

02.05 .01

Mitral valve - aortic valve discontinuity

02.05 .03

Mitral valve - pulmonary valve continuity

02.05 .02

Mitral valve - pulmonary valve discontinuity

02.05 .04

Mitral valve to arterial valve relationship unknown

02.05 .05

Relationship of RV with respect to LV, Either ventricle not apparent

02.04 .07

Relationship of RV with respect to LV, Left ventricle not apparent

02.04 .09

Relationship of RV with respect to LV, Right ventricle not apparent

02.04 .08

Relationship of RV with respect to LV, RV anterior with respect to LV

02.04 .05

Relationship of RV with respect to LV, RV inferior with respect to LV

02.04 .04

Relationship of RV with respect to $\mathrm{LV}, \mathrm{RV}$ posterior with respect to $\mathrm{LV}$

02.04 .06

Relationship of RV with respect to LV, RV superior with respect to LV

02.04 .03

Relationship of RV with respect to LV, RV to left with respect to LV

02.04 .02

Relationship of RV with respect to LV, RV to right with respect to LV

02.04 .01

Relationship of RV with respect to LV, Superior-inferior ventricular relationships

02.04 .00

Relationship of RV with respect to LV, Unknown

Relationships of great vessels-ascending aortic relationship, Parallel course of great arteries

02.04 .10

02.07 .01

Relationships of great vessels-ascending aortic relationship, Spiralling course of great arteries (normal)

02.07 .00

Relationships of great vessels-descending aortic relationship, Descending aorta and IVC same side with

03.08 .02

IVC anterior (Right isomerism)

Relationships of great vessels-descending aortic relationship, Descending aorta and vein same side with vein posterior (Left isomerism)

Relationships of great vessels-descending aortic relationship, Descending aorta left of spine and IVC right of spine (normal)

Relationships of great vessels-descending aortic relationship, Descending aorta right of spine and IVC left of spine (mirror image)

Ventricular imbalance

Ventricular imbalance, Dominant left ventricle + hypoplastic right ventricle

Ventricular imbalance, Dominant right ventricle + hypoplastic left ventricle

Ventricular topology

Ventricular topology anomaly

Ventricular topology, Left hand pattern ventricular topology (L-Loop)

Ventricular topology, Not possible to determine ventricular topology or unknown ventricular topology

Ventricular topology, Right hand pattern ventricular topology (D-Loop)

Ventricular topology, Solitary ventricle of indeterminate morphology

03.08 .03

03.08 .00

03.08.01

07.08.40

07.08 .41

07.08.42

Title

02.03.04

02.03 .02

02.03 .06

02.03 .01

02.03 .05

Syndrome, Heterotaxy (heterotaxy syndrome) (visceral heterotaxy)

03.01 .02

Syndrome, Heterotaxy (heterotaxy syndrome) (visceral heterotaxy) = Isomerism sequence (including asplenia, polysplenia, and Ivemark syndrome)

Syndrome, Heterotaxy (heterotaxy syndrome) (visceral heterotaxy), Ivemark syndrome (asplenia with visceroatrial heterotaxy and complex heart disease)

Syndrome, Heterotaxy (heterotaxy syndrome) (visceral heterotaxy)-modifier, Asplenia (spleen absent)

Syndrome, Heterotaxy (heterotaxy syndrome) (visceral heterotaxy)-modifier, Asplenia syndrome

Syndrome, Heterotaxy (heterotaxy syndrome) (visceral heterotaxy)-modifier, Bilateral bilobed (left) lungs (left isomerism)

Syndrome, Heterotaxy (heterotaxy syndrome) (visceral heterotaxy)-modifier, Bilateral left bronchi (long - left isomerism)

Syndrome, Heterotaxy (heterotaxy syndrome) (visceral heterotaxy)-modifier, Bilateral right bronchi (short - right isomerism)

Syndrome, Heterotaxy (heterotaxy syndrome) (visceral heterotaxy)-modifier, Bilateral trilobed (right) lungs (right isomerism)

Syndrome, Heterotaxy (heterotaxy syndrome) (visceral heterotaxy)-modifier, Intestines malrotated

Syndrome, Heterotaxy (heterotaxy syndrome) (visceral heterotaxy)-modifier, Isomerism

Syndrome, Heterotaxy (heterotaxy syndrome) (visceral heterotaxy)-modifier, Isomerism, Isomerism of the left atrial appendages

Syndrome, Heterotaxy (heterotaxy syndrome) (visceral heterotaxy)-modifier, Isomerism, Isomerism of the right atrial appendages

Syndrome, Heterotaxy (heterotaxy syndrome) (visceral heterotaxy)-modifier, Isomerism, Left isomerism

Syndrome, Heterotaxy (heterotaxy syndrome) (visceral heterotaxy)-modifier, Isomerism, Right isomerism 
Syndrome, Heterotaxy (heterotaxy syndrome) (visceral heterotaxy)-modifier, Polysplenia (multiple spleens) Syndrome, Heterotaxy (heterotaxy syndrome) (visceral heterotaxy)-modifier, Polysplenia syndrome Syndrome, Heterotaxy (heterotaxy syndrome) (visceral heterotaxy)-modifier, Situs ambiguus

Single ventricle, Heterotaxy (heterotaxy syndrome) (visceral heterotaxy)

Single ventricle, Heterotaxy (heterotaxy syndrome) (visceral heterotaxy), DORV + CAVC (CAVSD) + Asplenia (Right isomerism)

Single ventricle, Heterotaxy (heterotaxy syndrome) (visceral heterotaxy), DORV + CAVC (CAVSD) + Polysplenia (Left isomerism)

Single ventricle, Heterotaxy (heterotaxy syndrome) (visceral heterotaxy), Single LV

Systemic venous anomaly, IVC

Systemic venous anomaly, IVC, Abnormal RIVC

Systemic venous anomaly, IVC, Abnormal RIVC, Biatrial drainage of RIVC

Systemic venous anomaly, IVC, Abnormal RIVC, Biatrial drainage of RIVC, Hepatic veins to both atriums Systemic venous anomaly, IVC, Abnormal RIVC, Biatrial drainage of RIVC, Hepatic veins to CS

Systemic venous anomaly, IVC, Abnormal RIVC, Biatrial drainage of RIVC, Hepatic veins to left-sided atrium Systemic venous anomaly, IVC, Abnormal RIVC, Biatrial drainage of RIVC, Hepatic veins to right-sided atrium

Systemic venous anomaly, IVC, Abnormal RIVC, Interrupted RIVC

Systemic venous anomaly, IVC, Abnormal RIVC, Interrupted RIVC, Azygos continuation (absent suprarenal segment) to LSVC

Systemic venous anomaly, IVC, Abnormal RIVC, Interrupted RIVC, Azygos continuation (absent suprarenal segment) to LSVC, Hepatic veins to both atriums

Systemic venous anomaly, IVC, Abnormal RIVC, Interrupted RIVC, Azygos continuation (absent suprarenal segment) to LSVC, Hepatic veins to CS

Systemic venous anomaly, IVC, Abnormal RIVC, Interrupted RIVC, Azygos continuation (absent suprarenal segment) to LSVC, Hepatic veins to left-sided atrium

Systemic venous anomaly, IVC, Abnormal RIVC, Interrupted RIVC, Azygos continuation (absent suprarenal segment) to LSVC, Hepatic veins to right-sided atrium

Systemic venous anomaly, IVC, Abnormal RIVC, Interrupted RIVC, Azygos continuation (absent suprarenal segment) to RSVC

Systemic venous anomaly, IVC, Abnormal RIVC, Interrupted RIVC, Azygos continuation (absent suprarenal segment) to RSVC, Hepatic veins to both atriums

Systemic venous anomaly, IVC, Abnormal RIVC, Interrupted RIVC, Azygos continuation (absent suprarenal segment) to RSVC, Hepatic veins to CS

Systemic venous anomaly, IVC, Abnormal RIVC, Interrupted RIVC, Azygos continuation (absent suprarenal segment) to RSVC, Hepatic veins to left-sided atrium

Systemic venous anomaly, IVC, Abnormal RIVC, Interrupted RIVC, Azygos continuation (absent suprarenal segment) to RSVC, Hepatic veins to right-sided atrium

Systemic venous anomaly, IVC, Abnormal RIVC, Interrupted RIVC, Bi-Azygos continuation (absent suprarenal segment)

Systemic venous anomaly, IVC, Abnormal RIVC, Interrupted RIVC, Bi-Azygos continuation (absent suprarenal segment), Hepatic veins to both atriums

Systemic venous anomaly, IVC, Abnormal RIVC, Interrupted RIVC, Bi-Azygos continuation (absent suprarenal segment), Hepatic veins to CS

Systemic venous anomaly, IVC, Abnormal RIVC, Interrupted RIVC, Bi-Azygos continuation (absent suprarenal segment), Hepatic veins to left-sided atrium

Systemic venous anomaly, IVC, Abnormal RIVC, Interrupted RIVC, Bi-Azygos continuation (absent suprarenal segment), Hepatic veins to right-sided atrium

Systemic venous anomaly, IVC, Abnormal RIVC, RIVC to left-sided atrium

Systemic venous anomaly, IVC, Abnormal RIVC, RIVC to left-sided atrium, Hepatic veins to both atriums Systemic venous anomaly, IVC, Abnormal RIVC, RIVC to left-sided atrium, Hepatic veins to CS Systemic venous anomaly, IVC, Abnormal RIVC, RIVC to left-sided atrium, Hepatic veins to left-sided atrium Systemic venous anomaly, IVC, Abnormal RIVC, RIVC to left-sided atrium, Hepatic veins to right-sided atrium

Systemic venous anomaly, IVC, Biatrial drainage of IVC

Systemic venous anomaly, IVC, Cor triatriatum Dexter (Obstructive Eustachian valve)

Systemic venous anomaly, IVC, Interrupted IVC with azygos continuation (absent suprarenal segment)

Systemic venous anomaly, IVC, Interrupted IVC with azygos continuation (absent suprarenal segment), Left-sided azygos continuation of IVC to left SVC
03.07 .04

03.01 .05

01.03 .04

$01.01 .22,03.01 .02$

01.01.22, 03.01.02,

01.01.04, 06.06.00,

03.01 .04

01.01.22, 03.01.02,

01.01.04, 06.06.00, 03.01 .05

01.01.22, 03.01.02,

02.04 .08

04.03 .00

04.03 .15

04.03 .27

04.03.14, 04.02.03

$04.03 .14,04.02 .05$

$04.03 .14,04.02 .02$

$04.03 .14,04.02 .01$

04.03 .18

$04.03 .18,04.03 .19$

04.03.18, 04.03.19,

04.02 .03

04.03.18, 04.03.19,

04.02 .05

04.03.18, 04.03.19,

04.02 .02

04.03.18, 04.03.19,

04.02 .01

$04.03 .18,04.03 .20$

04.03.18, 04.03.20, 04.02 .03

04.03.18, 04.03.20, 04.02 .05

04.03.18, 04.03.20,

04.02 .02

04.03.18, 04.03.20,

04.02 .01

$04.03 .18,04.03 .03$

04.03.18, 04.03.03, 04.02 .03

04.03.18, 04.03.03, 04.02 .05

04.03.18, 04.03.03, 04.02 .02

04.03.18, 04.03.03, 04.02 .01

04.03 .04

$04.03 .04,04.02 .03$

$04.03 .04,04.02 .05$

04.03.04, 04.02.02

04.03.04, 04.02.01

04.03 .14

05.01 .21

04.03 .10

04.03 .01 
Systemic venous anomaly, IVC, Interrupted IVC with azygos continuation (absent suprarenal segment),

Right-sided azygos continuation of IVC to right SVC

Systemic venous anomaly, IVC, IVC connecting to coronary sinus

Systemic venous anomaly, IVC, LIVC

Systemic venous anomaly, IVC, LIVC, Biatrial drainage of LIVC

Systemic venous anomaly, IVC, LIVC, Biatrial drainage of LIVC, Hepatic veins to both atriums

Systemic venous anomaly, IVC, LIVC, Biatrial drainage of LIVC, Hepatic veins to CS

Systemic venous anomaly, IVC, LIVC, Biatrial drainage of LIVC, Hepatic veins to left-sided atrium

Systemic venous anomaly, IVC, LIVC, Biatrial drainage of LIVC, Hepatic veins to right-sided atrium

Systemic venous anomaly, IVC, LIVC, Interrupted LIVC

Systemic venous anomaly, IVC, LIVC, Interrupted LIVC, Azygos continuation (absent suprarenal segment) to LSVC

Systemic venous anomaly, IVC, LIVC, Interrupted LIVC, Azygos continuation (absent suprarenal segment) to LSVC, Hepatic veins to both atriums

Systemic venous anomaly, IVC, LIVC, Interrupted LIVC, Azygos continuation (absent suprarenal segment) to LSVC, Hepatic veins to CS

Systemic venous anomaly, IVC, LIVC, Interrupted LIVC, Azygos continuation (absent suprarenal segment) to LSVC, Hepatic veins to left-sided atrium

Systemic venous anomaly, IVC, LIVC, Interrupted LIVC, Azygos continuation (absent suprarenal segment) to LSVC, Hepatic veins to right-sided atrium

Systemic venous anomaly, IVC, LIVC, Interrupted LIVC, Azygos continuation (absent suprarenal segment) to RSVC

Systemic venous anomaly, IVC, LIVC, Interrupted LIVC, Azygos continuation (absent suprarenal segment) to RSVC, Hepatic veins to both atriums

Systemic venous anomaly, IVC, LIVC, Interrupted LIVC, Azygos continuation (absent suprarenal segment) to RSVC, Hepatic veins to CS

Systemic venous anomaly, IVC, LIVC, Interrupted LIVC, Azygos continuation (absent suprarenal segment) to RSVC, Hepatic veins to left-sided atrium

Systemic venous anomaly, IVC, LIVC, Interrupted LIVC, Azygos continuation (absent suprarenal segment) to RSVC, Hepatic veins to right-sided atrium

Systemic venous anomaly, IVC, LIVC, Interrupted LIVC, Bi-Azygos continuation (absent suprarenal segment) Systemic venous anomaly, IVC, LIVC, Interrupted LIVC, Bi-Azygos continuation (absent suprarenal segment), Hepatic veins to both atriums

Systemic venous anomaly, IVC, LIVC, Interrupted LIVC, Bi-Azygos continuation (absent suprarenal segment), Hepatic veins to CS

Systemic venous anomaly, IVC, LIVC, Interrupted LIVC, Bi-Azygos continuation (absent suprarenal segment), Hepatic veins to left-sided atrium

Systemic venous anomaly, IVC, LIVC, Interrupted LIVC, Bi-Azygos continuation (absent suprarenal segment),

Hepatic veins to right-sided atrium

Systemic venous anomaly, IVC, LIVC, LIVC to left-sided atrium

Systemic venous anomaly, IVC, LIVC, LIVC to left-sided atrium, Hepatic veins to both atriums

Systemic venous anomaly, IVC, LIVC, LIVC to left-sided atrium, Hepatic veins to CS

Systemic venous anomaly, IVC, LIVC, LIVC to left-sided atrium, Hepatic veins to left-sided atrium

Systemic venous anomaly, IVC, LIVC, LIVC to left-sided atrium, Hepatic veins to right-sided atrium

Systemic venous anomaly, IVC, LIVC, LIVC to right-sided atrium

Systemic venous anomaly, IVC, LIVC, LIVC to right-sided atrium, Hepatic veins to both atriums

Systemic venous anomaly, IVC, LIVC, LIVC to right-sided atrium, Hepatic veins to CS

Systemic venous anomaly, IVC, LIVC, LIVC to right-sided atrium, Hepatic veins to left-sided atrium

Systemic venous anomaly, IVC, LIVC, LIVC to right-sided atrium, Hepatic veins to right-sided atrium

Systemic venous anomaly, IVC, Separate entry of hepatic veins (RIVC to right-sided atrium)

Systemic venous anomaly, IVC, Separate entry of hepatic veins (RIVC to right-sided atrium), Hepatic veins to both atriums

Systemic venous anomaly, IVC, Separate entry of hepatic veins (RIVC to right-sided atrium), Hepatic veins to CS

Systemic venous anomaly, IVC, Separate entry of hepatic veins (RIVC to right-sided atrium), Hepatic veins to left-sided atrium

Systemic venous anomaly, IVC, Separate entry of hepatic veins (RIVC to right-sided atrium), Hepatic veins to right-sided atrium

Systemic venous anomaly, SVC

Systemic venous anomaly, SVC, Abnormal RSVC

Systemic venous anomaly, SVC, Abnormal RSVC, Bi-atrial drainage of RSVC

Systemic venous anomaly, SVC, Abnormal RSVC, RSVC to CS

Systemic venous anomaly, SVC, Abnormal RSVC, RSVC to CS (intact) to right-sided atrium

Systemic venous anomaly, SVC, Abnormal RSVC, RSVC to CS (partially unroofed)
04.03 .02

04.03 .05

04.03 .23

04.03 .24

$04.03 .24,04.02 .03$

$04.03 .24,04.02 .05$

04.03.24, 04.02.02

04.03.24, 04.02.01

04.03.25,

$04.03 .25,04.03 .19$

04.03.25, 04.03.19, 04.02 .03

04.03.25, 04.03.19, 04.02 .05

04.03.25, 04.03.19, 04.02 .02

04.03.25, 04.03.19, 04.02 .01

$04.03 .25,04.03 .20$

04.03.25, 04.03.20, 04.02 .03

$04.03 .25,04.03 .20$ 04.02 .05

04.03.25, 04.03.20, 04.02 .02

04.03.25, 04.03.20, 04.02 .01

$04.03 .25,04.03 .03$

04.03.25, 04.03.03, 04.02 .03

04.03.25, 04.03.03, 04.02 .05

04.03.25, 04.03.03, 04.02 .02

04.03.25, 04.03.03, 04.02 .01

04.03 .13

$04.03 .13,04.02 .03$

$04.03 .13,04.02 .05$

$04.03 .13,04.02 .02$

$04.03 .13,04.02 .01$

04.03 .12

$04.03 .12,04.02 .03$

$04.03 .12,04.02 .05$

$04.03 .12,04.02 .02$

$04.03 .12,04.02 .01$

$04.02 .06,04.00 .26$

04.02.06, 04.03.26, 04.02 .03

$04.02 .06,04.03 .26$ 04.02 .05

04.02.06, 04.03.26, 04.02 .02

04.02.06, 04.03.26, 04.02 .01

04.01 .00

04.01 .14

04.01 .15

04.01 .23

$04.01 .24,04.04 .16$

$04.01 .23,04.04 .01$ 
Systemic venous anomaly, SVC, Abnormal RSVC, RSVC to CS to left-sided atrium Systemic venous anomaly, SVC, Abnormal RSVC, RSVC to CS to left-sided atrium (completely unroofed CS)

Systemic venous anomaly, SVC, Abnormal RSVC, RSVC to left-sided atrium

Systemic venous anomaly, SVC, Absent RSVC

Systemic venous anomaly, SVC, Absent RSVC, LSVC present

Systemic venous anomaly, SVC, Absent RSVC, LSVC present, Bi-atrial drainage of LSVC

Systemic venous anomaly, SVC, Absent RSVC, LSVC to CS

Systemic venous anomaly, SVC, Absent RSVC, LSVC to CS (intact) to right-sided atrium

Systemic venous anomaly, SVC, Absent RSVC, LSVC to CS (partially unroofed)

Systemic venous anomaly, SVC, Absent RSVC, LSVC to CS to right-sided atrium

Systemic venous anomaly, SVC, Absent RSVC, LSVC to left-sided atrium

Systemic venous anomaly, SVC, Absent RSVC, LSVC to left-sided atrium

(completely unroofed CS)

Systemic venous anomaly, SVC, Absent RSVC, LSVC to right-sided atrium

Systemic venous anomaly, SVC, Atretic LSVC

Systemic venous anomaly, SVC, Atretic RSVC

Systemic venous anomaly, SVC, Bilateral SVC

Systemic venous anomaly, SVC, Bilateral SVC, Innominate absent

Systemic venous anomaly, SVC, Bilateral SVC, Innominate present

Systemic venous anomaly, SVC, Bilateral SVC, LSVC to CS (intact) to right-sided atrium

Systemic venous anomaly, SVC, Bilateral SVC, LSVC to CS (intact) to right-sided atrium, Innominate absent

Systemic venous anomaly, SVC, Bilateral SVC, LSVC to CS (intact) to right-sided atrium, Innominate present

Systemic venous anomaly, SVC, Bilateral SVC, LSVC to CS (partially unroofed)

Systemic venous anomaly, SVC, Bilateral SVC, LSVC to CS (partially unroofed), Innominate absent

Systemic venous anomaly, SVC, Bilateral SVC, LSVC to CS (partially unroofed), Innominate present

Systemic venous anomaly, SVC, Bilateral SVC, LSVC to left-sided atrium (completely unroofed CS)

Systemic venous anomaly, SVC, Bilateral SVC, LSVC to left-sided atrium (completely unroofed CS), Innominate absent

Systemic venous anomaly, SVC, Bilateral SVC, LSVC to left-sided atrium (completely unroofed CS), Innominate present

Systemic venous anomaly, SVC, Bilateral SVC, RSVC to CS to left-sided atrium

Systemic venous anomaly, SVC, Bilateral SVC, RSVC to CS to left-sided atrium, Innominate absent

Systemic venous anomaly, SVC, Bilateral SVC, RSVC to CS to left-sided atrium, Innominate present

Systemic venous anomaly, SVC, CS ostial atresia or stenosis (CS draining cephalad via LSVC)

Systemic venous anomaly, SVC, CS ostial atresia or stenosis (CS draining cephalad via LSVC),

CS ostial atresia

Systemic venous anomaly, SVC, CS ostial atresia or stenosis (CS draining cephalad via LSVC),

CS ostial stenosis

Systemic venous anomaly, SVC, CS ostial atresia or stenosis (CS draining cephalad via RSVC)

Systemic venous anomaly, SVC, CS ostial atresia or stenosis (CS draining cephalad via RSVC),

CS ostial atresia

Systemic venous anomaly, SVC, CS ostial atresia or stenosis (CS draining cephalad via RSVC),

CS ostial stenosis

Systemic venous anomaly, SVC, Levoatrial-cardinal vein (Persistent left posterior cardinal vein)

(LA to innominate vein)

Systemic venous anomaly, SVC, Retro-aortic Innominate vein
04.01 .12

$04.01 .12,04.04 .02$

04.01 .10

04.01.05

$04.01 .05,04.01 .25$

$04.01 .05,04.01 .16$

$04.01 .05,04.01 .01$

04.01.05, 04.01.26, 04.04 .16

04.01.05, 04.01.01, 04.04 .01

$04.01 .05,04.01 .26$

$04.01 .05,04.01 .02$

04.01.05, 04.01.02,

04.04 .02

04.01.05, 04.01.13

04.01 .19

04.01 .04

04.01 .03

$04.01 .03,04.05 .01$

$04.01 .03,04.01 .18$

04.01.03, 04.01.26, 04.04 .16

04.01.03, 04.01.26,

04.04 .1604 .05 .01

04.01.03, 04.01.26,

$04.04 .16,04.01 .18$

$04.01 .03,04.01 .01$

04.04 .01

04.01.03, 04.01.01

04.04.01, 04.05.01

04.01.03, 04.01.01

04.04.01, 04.01.18

$04.01 .03,04.01 .02$,

04.04 .02

04.01.03, 04.01.02,

04.04.02, 04.05.01

04.01.03, 04.01.02,

$04.04 .02,04.01 .18$

$04.01 .03,04.01 .12$

04.01.03, 04.01.12, 04.05 .01

04.01.03, 04.01.12,

04.01 .18

04.04.14, 04.04.11

$04.04 .03,04.04 .11$

04.04.04, 04.04.11

$04.04 .14,04.04 .10$

$04.04 .03,04.04 .10$

04.04.04, 04.04.10

04.01 .06

04.00 .04

Abbreviations: AV, atrioventricular; CAVC (CAVSD), complete atrioventricular canal (complete atrioventricular septal defect); CS, coronary sinus; DORV, double outlet right ventricle; IVC, inferior vena cava; IVS, intact ventricular septum; LA, left atrium; LIVC, left inferior vena cava; LSVC, left superior vena cava; LV, left ventricle; RIVC, right inferior vena cava; RSVC, right superior vena cava; RV, right ventricle; SVC, superior vena cava; TGA, transposition of the great arteries; TOF, tetralogy of Fallot, VA, ventriculoarterial; VSD, ventricular septal defect. 
Table 2. The Association for European Paediatric Cardiology derived version of the International Paediatric and Congenital Cardiac Code for the diagnoses related to heterotaxy.

Normal heart

01.01 .00

Normal atrial arrangement (situs), AV \& VA connections

01.03 .10

Systemic venous connections normal

04.00 .00

Pulmonary venous connections normal

04.00 .03

Position and orientation of heart

Normal position/orientation of heart

02.01 .00

Position-orientation of heart abnormal

02.01 .09

Laevocardia: heart predominantly in left hemithorax

02.01 .03

Heart rotated to left (laevorotation)

02.01 .06

Dextrocardia: heart predominantly in right hemithorax

02.01 .02

Heart rotated to right (dextrorotation)

02.01 .05

Midline heart (mesocardia)

02.01 .04

Apex of heart to left ("laevoversion")

02.02 .00

Apex of heart to right ("dextroversion")

02.02 .01

Apex of heart anterior to the cardiac base

02.02 .20

Apex of heart posterior to the cardiac base

02.02 .21

Bifid apex of heart

02.02 .03

Midline apex of heart

02.02 .04

Midline apex of heart: anterior

02.02 .18

Midline apex of heart: posterior

02.02 .19

Isomerism and non-cardiac organs

Isomerism

01.03 .12

Visceral heterotaxy (abnormal arrangement thoraco-abdominal organs)

03.01 .02

Isomerism sequence (including asplenia/polysplenia \& Ivemark syndromes)

14.02 .03

Ivemark syndrome (asplenia with right isomerism \& complex congenital heart disease)

14.02 .95

Right isomerism ("asplenia")

Left isomerism ("polysplenia")

03.01 .04

03.01 .05

Bilateral trilobed (right) lungs (right isomerism)

03.02 .03

Bilateral bilobed (left) lungs (left isomerism)

03.02 .04

Bilateral right bronchi (short - right isomerism)

03.03 .03

Bilateral left bronchi (long - left isomerism)

03.03 .04

Spleen absent (asplenia)

03.07 .03

Multiple spleens (polysplenia)

03.07.04

Liver centrally situated

03.04 .03

Intestines malrotated

03.06 .03

Atrial arrangements (situs)

Usual atrial arrangement (atrial situs solitus)

01.03 .00

Abnormal atrial arrangement

01.03 .06

Mirror image atrial arrangement (atrial situs inversus)

01.03 .01

Atrial arrangement (situs) ambiguus (indeterminate)

01.03 .04

Isomerism of atrial appendages

Isomerism of right atrial appendages (right isomerism)

01.03 .08

Isomerism of left atrial appendages (left isomerism)

01.03 .02

Atrial arrangement unknown

01.03 .03

$\mathrm{AV}$ and/or VA connections abnormal

01.03 .07

01.03 .09

Atrioventricular (AV) connections

Concordant AV connections

01.04 .00

Discordant AV connections

01.04 .01

Mixed ('ambiguous') AV connections (biventricular)

01.04 .02

Absent AV connection (single inlet ventricle)

01.04 .21

Absent right-sided AV connection

01.04 .19

Absent right-sided AV connection (univentricular)

01.04 .12

Left-sided atrium to left ventricle

01.04 .06

Left-sided atrium to right ventricle

01.04 .07

Left-sided atrium to both ventricles

01.04 .17

Left-sided atrium to ventricle of indeterminate morphology

01.04 .08

Absent right-sided AV connection with straddling valve (uniatrial biventricular)

01.04 .15

Absent left-sided AV connection

01.04 .20

Absent left-sided AV connection (univentricular)

01.04 .13

Right-sided atrium to right ventricle

01.04 .09

Right-sided atrium to left ventricle

01.04 .10 
Continued

Right-sided atrium to both ventricles

01.04 .18

Right-sided atrium to ventricle of indeterminate morphology

01.04 .11

Absent left-sided AV connection with straddling valve (uniatrial biventricular)

01.04 .16

Double inlet AV connection (double inlet ventricle)

01.01 .14

Double inlet right ventricle

01.04 .03

Double inlet left ventricle

01.04 .04

Double inlet to solitary ventricle of indeterminate morphology

01.04 .05

Two AV valves in double inlet ventricle

Common AV orifice in double inlet ventricle

01.06 .01

01.06 .02

Mode of AV connection unknown

01.04 .23

Atrioventricular connection(s) unknown

01.04 .22

Criss-cross heart (twisted AV connections)

02.03 .03

Criss-cross heart with leftward rotation

02.03 .11

Criss-cross heart with rightward rotation

02.03 .12

Ventriculo-arterial (VA) connections

Concordant VA connections

01.05 .00

Concordant VA connections with parallel great arteries (anatomically corrected malposition)

01.05 .10

Discordant VA connections (TGA)

Double outlet VA connections

01.05 .01

01.01 .50

Double outlet right ventricle

Double outlet right ventricle: transposition type (subpulmonary VSD)

01.01 .04

01.01 .18

01.01 .17

01.01 .40

Double outlet right ventricle: subaortic or doubly committed VSD without pulmonary stenosis ("VSD type")

01.01 .19

Double outlet right ventricle: with doubly committed subarterial VSD

01.01 .23

Double outlet right ventricle: with intact ventricular septum

01.01 .24

01.05 .03

Double outlet left ventricle

Double outlet from ventricle of indeterminate morphology

01.05 .04

Single outlet VA connection

Common arterial trunk (truncus arteriosus)

Single outlet VA connection via aorta (pulmonary atresia)

Single outlet VA connection via pulmonary trunk (aortic atresia)

01.01 .51

09.01 .01

01.05 .32

01.05 .33

09.07 .26

Solitary arterial trunk (absent intrapericardial pulmonary arteries)

01.05 .41

01.05 .42

Single ventricular outlet above right ventricle

01.05 .43

Single ventricular outlet above ventricle of indeterminate morphology

01.05 .44

Ventricular commitment of VA artery unknown or unspecified

Ventriculo-arterial connection(s) unknown or unspecified

01.05 .46

VSD in double outlet VA connection

VSD in double outlet VA connection - doubly committed

01.05 .17

07.13 .01

07.13 .02

VSD in double outlet VA connection - non-committed

07.13 .03

VSD in double outlet VA connection - subaortic

07.13 .04

VSD in double outlet VA connection - subpulmonary

07.13 .05

Cardiac topology and relationships

Right hand pattern ventricular topology

02.03 .01

Left hand pattern ventricular topology

02.03 .02

Ventricular topology pattern not determinable

02.03 .06

Ventricular topology anomaly

02.03 .04

02.03 .05

Solitary ventricle of indeterminate morphology

02.04 .01

Right ventricle to

02.04 .02

02.04 .03

Right ventricle superior with respect to left ventricle

02.04 .04

Right ventricle inferior with respect to left ventricle

02.04 .05

Right ventricle anterior with respect to left ventricle

02.04 .06

Superior-inferior ("upstairs-downstairs") ventricular relationship

02.04 .00

Right ventricle not apparent

02.04 .08

Left ventricle not apparent

02.04 .09

Either ventricle not apparent

02.04 .07

Ventricular relationships unknown

02.04 .10

Functionally univentricular heart

01.01 .22 
Ventricular imbalance

07.08 .40

Ventricular imbalance: dominant left ventricle + hypoplastic right ventricle

Ventricular imbalance: dominant right ventricle + hypoplastic left ventricle

Mitral valve - aortic valve continuity

02.05 .01

Mitral valve - pulmonary valve continuity

Mitral valve - aortic valve discontinuity

02.05 .03

Mitral valve - pulmonary valve discontinuity

02.05 .04

Mitral valve - arterial valve relationship unknown

Aortic orifice posterior right with respect to pulmonary orifice (normal)

Aortic orifice right side-by-side with respect to pulmonary orifice

02.06 .01

Aortic orifice anterior right with respect to pulmonary orifice

02.06 .02

Aortic orifice anterior with respect to pulmonary orifice

02.06 .03

Aortic orifice anterior left with respect to pulmonary orifice

02.06 .04

Aortic orifice left side-by-side with respect to pulmonary orifice

02.06 .05

Aortic orifice posterior left with respect to pulmonary orifice

02.06 .06

Aortic orifice posterior with respect to pulmonary orifice

02.06 .07

Aortic orifice to pulmonary orifice relationship unknown

02.06 .11

Abnormal relationship of aortic orifice to pulmonary orifice

02.06 .09

Aortic sinuses malaligned with respect to pulmonary sinuses

Descending aorta left of spine and inferior caval vein (IVC) right (normal)

03.08 .00

Descending aorta right of spine and inferior caval vein (IVC) left (mirror image)

03.08 .01

Descending aorta and inferior caval vein (IVC) same side with inferior caval vein (IVC) anterior (right isomerism)

03.08 .02

Descending aorta and vein same side with vein posterior (left isomerism)

03.08 .03

09.28 .01

Left arch \& left descending aorta (normal)

09.28 .22

Left aortic arch branching pattern

Abnormal course of aortic arch

09.30 .25

09.28 .23

Abnormal course of aortic arch and descending aorta

09.28 .19

Left arch \& right descending aorta

09.28 .02

Right aortic arch

09.28 .15

Right aortic arch (mirror image) branching pattern

09.30 .26

Right arch \& right descending aorta

09.28 .03

Right arch \& left descending aorta

09.28 .04

Aortic arch centrally descending

Anomalies of AV valves associated with heterotaxy

Imperforate $\mathrm{AV}$ valve

06.00 .07

Tricuspid valve atretic (imperforate)

06.01 .02

Mitral valve atretic (imperforate)

Imperforate right-sided AV valve

06.02 .02

Imperforate left-sided $A V$ valve

06.00 .12

Overriding AV valve

Overriding tricuspid valve

06.00 .13

06.00 .01

06.01 .05

Overriding mitral valve

06.02 .05

Overriding right-sided AV valve

06.00 .02

Overriding left-sided AV valve

06.00 .02

- override of AV valve $<50 \%$

Q1.27.74

Q1.27.75

- override of AV valve $50-90 \%$

Q1.27.76

- override of AV valve $>90 \%$

06.00 .04

Straddling AV valve

06.01 .09

Straddling tricuspid valve

Straddling right-sided AV valve

Straddling left-sided AV valve

06.00 .06

Atrioventricular septal defect

Anomalies of VA valves associated with heterotaxy 
Continued

Aortic valvar atresia - imperforate valve

09.15 .04

Imperforate right-sided VA valve

09.04 .26

Imperforate left-sided VA valve

09.04 .25

Overriding VA valve

Pulmonary valve overriding ventricular septum

09.04 .21

Aortic valve overriding ventricular septum

09.05 .52

Overriding right-sided VA valve

09.15 .47

Overriding left-sided VA valve

09.04 .23

- override of VA valve $<50 \%$

09.04 .24

- override of VA valve $50-90 \%$

Q1.27.98

Q1.27.99

- override of VA valve $>90 \%$

Q1.28.00

Anomalies of great veins and coronary sinus associated with heterotaxy

Inferior caval vein (IVC) abnormality

04.03 .00

Inferior caval vein (IVC) connecting to both atriums

Inferior caval vein (IVC) interruption (absent suprarenal segment) with azygos continuation

Left-sided azygos continuation of inferior caval vein (IVC) (absent suprarenal segment) to left superior caval vein (SVC)

04.03 .14

04.03 .10

Right-sided azygos continuation of inferior caval vein (IVC) (absent suprarenal segment) to right superior caval vein (SVC)

04.03 .01

Inferior caval vein (IVC) interruption (absent suprarenal segment) with bilateral azygos continuation

04.03 .02

04.03 .03

Azygos continuation of inferior caval vein (IVC) (absent suprarenal segment) to left superior caval vein (SVC)

Azygos continuation of inferior caval vein (IVC) (absent suprarenal segment) to right superior caval vein (SVC)

04.03 .19

Inferior caval vein (IVC) connecting to coronary sinus

04.03 .20

04.03 .05

Right inferior caval vein (IVC) abnormality,

04.03 .15

Right inferior caval vein (IVC) connecting to right-sided atrium

04.03 .26

Right inferior caval vein (IVC) connecting to left-sided atrium

04.03 .04

Right inferior caval vein (IVC) connecting to both atriums

04.03 .27

Interrupted right inferior caval vein (IVC)

Left inferior caval vein (IVC)

Left inferior caval vein (IVC) connecting to right-sided atrium

04.03 .18

04.03 .16

Left inferior caval vein (IVC) connecting to left-sided atriu

04.03 .12

04.13 .13

Left inferior caval vein (IVC) connecting to both atriums

04.03 .24

Interrupted left inferior caval vein (IVC)

Obstructive Eustachian valve (divided right atrium) (cor triatriatum dexter)

04.03 .25

05.01 .21

Superior caval vein (SVC) abnormality,

04.01 .00

Right superior caval vein (SVC) abnormality,

04.01 .14

Right superior caval vein (SVC) connecting to left-sided atrium

04.01 .10

Right superior caval vein (SVC) connecting to both atriums

04.01 .15

Right superior caval vein (SVC) connecting to coronary sinus

04.01 .23

04.01 .24

Right superior caval vein (SVC) connecting to coronary sinus to right-sided atrium

04.01 .12

Absent right superior caval vein (SVC)

Atretic right superior caval vein (SVC

04.01 .05

04.01 .04

Left superior caval vein (SVC)

Left superior caval vein (SVC) persisting to coronary sinus

04.01 .25

04.01 .01

Left superior caval vein (SVC) persisting to coronary sinus to right-sided atrium

04.01 .26

Left superior caval vein (SVC) connecting to right-sided atrium

04.01 .13

Left superior caval vein (SVC) persisting to left-sided atrium

04.01 .02

Left superior caval vein (SVC) connecting to both atriums

04.01 .16

04.01 .19

Atretic left superior caval vein (SVC)

Bilateral superior caval veins (SVC)

04.01 .03

Innominate vein present

Absent bridging (innominate) vein

04.01 .18

04.05 .01

Retro-aortic innominate vein

04.00 .04

Persistent left posterior (laevo-atrial) cardinal vein (left atrium to innominate vein)

04.01 .06

Separate hepatic venous and inferior caval venous connections to heart

04.02 .06

Hepatic veins to right-sided atrium

04.02 .01

Hepatic veins to left-sided atrium

04.02 .02

Hepatic veins to bilateral atriums

04.02 .03

Hepatic veins to coronary sinus

04.02 .05

Coronary sinus intact

04.04 .16

Coronary sinus defect in left atrium: partially unroofed

04.04 .01 
Abbreviations: AV, Atrioventricular; IVC, inferior vena cava; SVC, superior vena cava, VA, ventriculo-arterial; VSD, ventricular septal defect.

\section{Conclusions}

We have proposed that heterotaxy be defined to encompass the group of abnormalities in which the internal thoraco-abdominal organs, including the atrial appendages, demonstrate abnormal arrangement across the left-right axis of the body. The heterotaxy syndrome as thus defined is typically associated with complex cardiovascular malformations. Proper description of the heart in patients with this syndrome requires complete description of both the cardiac relations and the junctional arrangement of the cardiac segments, with documentation of the arrangement of the atrial appendages, the ventricular topology, the nature of the unions of the segments across the atrioventricular and the ventriculoarterial junctions, the infundibular morphologies, and the relationships of the arterial trunks in space. The position of the heart in the chest, and the orientation of the cardiac apex, must also be described separately. Particular attention is required for the venoatrial connections, since these are so often abnormal. The malformations within the heart are then analysed and described separately as for any patient with suspected congenital cardiac disease. The relationship and arrangement of the remaining thoraco-abdominal organs, including the lungs, the spleen, and the intestines, also must be described separately, because, although common patterns of association have been identified, there are frequent exceptions to these common patterns. It is only by utilizing this stepwise and logical progression of analysis that it becomes possible to describe correctly, and to classify properly, patients with heterotaxy. Although the degree of certainty in classifying the patients with heterotaxy may be very high when based on examination of pathological specimens, at times it remains a challenge in live patients, despite the best currently available diagnostic modalities. It is possible that in the future, genetic phenotyping may become of value in helping the clinician overcome this challenge. Although a few issues remain contentious, the Nomenclature Working Group has achieved a high degree of consensus on most of the issues discussed in this review.

\section{Acknowledgements}

We thank the Children's Heart Foundation (http:// www.childrensheartfoundation.org/) for financial support of this research. We acknowledge in particular the contributions of several investigators who contributed significantly:

- Tina Merola, Tracey Cox, and Debbie McIntosh of The Congenital Heart Institute of Florida, Cardiac Surgical Associates, All Children's Hospital, Children's Hospital of Tampa, and the University of South Florida, Saint Petersburg and Tampa, Florida, United States of America

- Janet Kreutzer, of The Congenital Heart Institute of Florida, Cardiac Surgical Associates, Children's Hospital of Tampa, and the University of South Florida, Saint Petersburg and Tampa, Florida, United States of America

- Jean Wilhelm of The Congenital Heart Institute of Florida, Cardiac Surgical Associates, All Children's Hospital, and the University of South Florida, Saint Petersburg and Tampa, Florida, United States of America

We also thank the following organizations for their generous support:

- American College of Cardiology

- Andy Collins for Kids Fund

- Council on Cardiovascular Disease in the Young of the American Heart Association

- The Heart and Stroke Foundation of Canada

- Institute of Circulatory and Respiratory Health of the Canadian Institutes of Health Research

- The Montreal Children's Hospital Foundation

- Japan Research Promotion Society for Cardiovascular Diseases

- Tokyo Women's Medical University

\section{References}

1. Jacobs JP, Franklin RCG, Jacobs ML, et al. Classification of the functionally univentricular heart: unity from mapped codes. Cardiol Young 2006; 16 (1 Suppl): 9-21. 
2. Tchervenkov CI, Jacobs JP, Weinberg PM, et al. The nomenclature, definition and classification of hypoplastic left heart syndrome. Cardiol Young 2006; 16: 339-368.

3. Jacobs JP, Franklin RCG, Wilkinson JL, et al. The nomenclature, definition and classification of discordant atrioventricular connections. Cardiol Young 2006; 16 (3 Suppl): 72-84.

4. Van Praagh R. The segmental approach to diagnosis in congenital heart disease. Birth Defects original article series 1972; 8: 4-23.

5. Van Praagh RTerminology of congenital heart disease: glossary and commentary, Circulation 1977; 56: 139-143.

6. Van Praagh R, Vlad P. Dextrocardia, mesocardia, and levocardia: the segmental approach in congenital heart disease. In: Keith JD, Rowe RD, Vlad P (eds). Heart disease in infancy and childhood, 3rd edn. Macmillan, New York, 1978, pp ....

7. Anderson RH, Becker AE, Freedom RM, et al. Sequential segmental analysis of congenital heart disease. Pediatr Cardiol 1984; 5: 281-287.

8. Wilcox BR, Anderson RH. Surgical anatomy of the heart, 2nd edn. Gower Medical Publishing, London, 1992.

9. Anderson RH, Ho SY. Sequential segmental analysis - description and catergorization for the millenium. Cardiol Young 1997; 7: 98-116.

10. Van Mierop LHS, Gessner IH, Schiebler GL. Asplenia and polysplenia syndromes. In: Bergsma D (ed). Birth defects: atlas and compendium. Williams \& Wilkins, Baltimore, 1972, pp ....

11. Macartney FJ. Classification and nomenclature of congenital heart defects. In: Stark J, deLeval M (eds). Surgery for congenital heart defects, 2nd edn. WB Saunders, Philadelphia, 1994, pp ....

12. Jacobs JP. Nomenclature and classification for congenital cardiac surgery. In: Mavroudis C, Backer CL (eds). Pediatric Cardiac Surgery, 3rd edn. Mosby Inc., An affiliate of Elsevier, Philadelphia, Pennsylvania, 2003, pp 25-38.

13. Fontana GP, Burke RP. Straddling and overriding atrioventricular valves. In: Baue AE, Geha AS, Hammond GL, et al (eds). Glenn's Thoracic and Cardiovascular Surgery, 6th edn. Appleton \& Lange, Stamford, Conn, 1996, pp ...

14. Van Praagh R. Cardiac anatomy. In: Chang AC, Hanley FL, Wernovsky G, Wessel DL (eds). Pediatric Cardiac Intensive Care, Williams and Wilkins, Baltimore, Maryland, 1998, pp 3-15.

15. Huhta JC, Smallhorn JF, Macartney FJ. Two dimensional echocardiographic diagnosis of situs. Br Heart J 1982; 48: 97-108.

16. Van Praagh R, David I, Wright GB, Van Praagh S. Large RV plus small LV is not single LV. Circulation 1980; 61: 1057-1058.

17. Keeton BR, Macartney FJ, Hunter S, et al. Univentricular heart of right ventricular type with double or common inlet. Circulation 1979; 59: 403-411.

18. Uemura H, Ho SY, Devine WA, Kilpatrick LL, Anderson RH. Atrial appendages and venoatrial connections in hearts with patients with visceral heterotaxy. Ann Thorac Surg 1995; 60: 561-569.

19. Van Mierop LHS, Wiglesworth FW. Isomerism of the cardiac atria in the asplenia syndrome. Lab Invest 1964; 11: 1303-1315.

20. Van Mierop LHS, Patterson PR, Reynolds RW. Two cases of congenital asplenia with isomerism of the cardiac atria and the sinoatrial nodes. Am J Cardiol 1964; 13: 407-412.

21. Moller JH, Nakib A, Anderson RC, Edwards JE. Congenital cardiac disease associated with polysplenia: a developmental complex of bilateral "left-sidedness". Circulation 1967; 36: 789-799.

22. Van Praagh R, Van Praagh S. Atrial isomerism in the heterotaxy syndromes with asplenia, or polysplenia, or normally formed spleen: an erroneous concept. Am J Cardiol 1990; 66: 1504-1506.

23. Liu C, Liu W, Lu M-F, Brown NA, Martin JF. Regulation of leftright asymmetry by thresholds of Pitx2 activity. Development 2001; 128: 2039-2048.
24. Bamforth SD, Bragança J, Farthing CR, et al. Cited2 controls left-right patterning and heart development through a NodalPitx2c pathway. Nat Genet 2004; 36: 1189-1196.

25. Schilling TF, Concorder JP, Ingham PW. Regulation of left-right asymmetries in the zebrafish by shh and BMP4. Dev Biol 1999; 210: 277-287.

26. Macartney FJ, Anderson RH, Smallhorn JF, et al. Segmental analysis in practice. In: Becker AE, Marceletti C, Losekoot TG (eds). Paediatric Cardiology Volume 3, Churchill Livingstone, Edinburgh, 1980, pp ...

27. Macartney FJ, Partridge JB, Shinebourne EA, et al. Identification of atrial situs. In: Anderson RH, Shinebourne EA (eds). Paediatric Cardiology 1977. Churchill Livingstone, Edinburgh, 1978, pp ...

28. Van Mierop LH, Eisen S, Schiebler GL. The radiographic appearance of the tracheobronchial tree as an indicator of the visceral situs. Am J Cardiol 1970; 26: 432-435.

29. Partridge JB, Scott O, Deverall PB, Macartney FJ. Visualization and measurement of the main bronchi by tomography as an objective indicator of thoracic situs in congenital heart disease. Circulation 1975; 51: 188-196.

30. Brandt PW, Calder AL. Cardiac connections: the segmental approach to radiologic diagnosis in congenital heart disease. Curr Probl Diagn Radiol 1977; 7: 1-35.

31. Stanger P, Rudolph AM, Edwards JE. Cardiac malpositions: an overview based on study of sixty-five necropsy specimens. Circulation 1977; 56: 159-172.

32. Caruso G, Becker AE. How to determine atrial situs? Considerations initiated by 3 cases of absent spleen with a discordant anatomy between bronchi and atria. Br Heart J 1979; 41: 559-567.

33. Landing BH, Lawrence TY, Payne Jr. VC, Wells TR. Bronchial anatomy in syndromes with abnormal visceral situs, abnormal spleen and congenital heart disease. Am J Cardiol 1971; 28: 456-462.

34. Layman TE, Levine MA, Amplatz K, Edwards JE. "Asplenic syndrome" in association with rudimentary spleen. Am J Cardiol 1967; 20: 136-140.

35. Walters 3rd HW, Mavroudis C, Tchervenkov CI, Jacobs JP, Lacour-Gayet F, Jacobs ML. Congenital Heart Surgery Nomenclature and Database Project: double outlet right ventricle. Ann Thorac Surg 2000; 69 (Suppl): S249-263.

36. Tchervenkov CI, Walters 3rd. HW, Chu VFCongenital Heart Surgery Nomenclature and Database Project: double outlet left ventricle, Ann Thorac Surg 2000; 69 (Suppl): S264-269.

37. Van Praagh R, Van Praagh S, Vlad P, Keith JD. Anatomic types of congenital dextrocardia. Diagnostic and embryologic implications. Am J Cardiol 1964; 13: 510-531.

38. Shinebourne EA, Macartney FJ, Anderson RH. Sequential chamber localisation. Br Heart J 1976; 38: 327-340.

39. Anderson RH, Smith A, Wilkinson JL. Disharmony between atrioventricular connections and segmental combinations: unusual variants of "crisscross" hearts. JACC 1987; 10: 1274-1277.

40. Wilkinson JL, Cochrane AD, Karl TRCongenital Heart Surgery Nomenclature and Database Project: corrected (discordant) transposition of the great arteries (and related malformations), Ann Thorac Surg 2000; 69 (Suppl): S236-248.

41. Wilkinson JL, Acerete F. Terminological pitfalls in congenital heart disease. Reappraisal of some confusing terms, with an account of a simplified system of basic nomenclature. Br Heart J 1973; 35: 1166-1177. PMID: 4761119 [PubMed - indexed for MEDLINE].

42. Macartney FJ, Zuberbuhler JR, Anderson RH. Morphological considerations pertaining to recognition of atrial isomerism. Consequences for sequential chamber localisation. Br Heart J 1980; 44: 657-667.

43. Uemura H, Ho SY, Anderson RH, et al. The surgical anatomy of coronary venous return in hearts with isomeric atrial appendages. J Thorac Cardiovasc Surg 1995; 110: 436-444. 
44. Uemura H, Ho SY, Devine WA, Anderson RH. Analysis of visceral heterotaxy according to splenic status, appendage morphology, or both. Am J Cardiol 1995; 76: 846-849.

45. Van Praagh S, Kreutzer J, Van Praagh R. Systemic and pulmonary venous connections in visceral heterotaxy, with emphasis on the diagnosis of the atrial situs: a study of 109 postmortem cases. In: Clark EB, Takao A (eds). Developmental Cardiology: Morphogenesis and Function, 1st edn. Futura Publishing Co., Inc., Mount Kisco, NY, 1990, pp 671-727.

46. Zhu L. Belmont JW. Ware SM. Genetics of human heterotaxias. Eur J Hum Genet 2006; 14: 17-25.

47. Berg C, Geipel A, Kamil D, et al. The syndrome of right isomerism - prenatal diagnosis and outcome. Ultraschall in der Medizin 2006; 27: 225-233.

48. Solomons TWG (University of South Florida). Organic Chemistry, 2nd edn. John Wiley and Sons, Inc., New York, 1980, pp 282-283.
49. Bartram U, Johannes Wirbelauer J, Speer CP. Heterotaxy syndrome - asplenia and polysplenia as indicators of visceral Mmlposition and complex congenital heart disease. Biol Neonate 2005; 88: 278-290. DOI: 10.1159/000087625

50. Ivemark BI. Implications of agenesis of the spleen on the pathogenesis of conotruncus anomalies in childhood; an analysis of the heart malformations in the splenic agenesis syndrome, with fourteen new cases. Acta Paediatr Suppl 1955; 44 (104 Suppl): 7-110.

51. http://children.webmd.com/Ivemark-Syndrome, accessed April 8, 2007.

52. Franklin RCG, Jacobs JP, Tchervenkov CI, Béland M. Bidirectional crossmap of the Short Lists of the European Paediatric Cardiac Code and the International Congenital Heart Surgery Nomenclature and Database Project. Cardiol Young 2002; 12 (II Suppl): $18-22$. 


\section{Appendix}

When discussing the morphology of the atriums and the ventricles, and their spatial relationships, the words "left" and "right" can be confusing. ${ }^{52}$ Rules are required, therefore, to provide consistency and accuracy when describing anatomical phenotypes. ${ }^{52}$ For cardiac chambers, unless otherwise stated, "left" refers to those chambers that, in the normal individual, are positioned on the left side of the body, and "right" to those that, in the normal person, are right-sided. To provide specificity, however, it is better to describe the chambers as being "morphologically left" or "morphologically right". Thus, in the patient with a congenitally malformed heart, irrespective of whether the observer uses the European system, or that derived from the teachings of Van Praagh, left ventricle means the morphologically left ventricle, left atrium refers to the morphologically left atrium, and right atrial appendage refers to the morphologically right atrial appendage, and so on. When discussing cardiac chambers, therefore, the words "left" and "right", if used in isolation, should not imply sidedness or position. When describing the position, or sidedness, of a cardiac chamber, terms such as "left-sided ventricle" should be used. The default term of left ventricle, therefore, at least for those describing congenitally malformed hearts, should mean the morphologically left ventricle, and should not mean or imply left-sidedness or right-sidedness. Similarly, it does not imply junctional union with either the right or left atrium, or the pulmonary or systemic circulations. In contrast, when describing the superior caval vein, and using the prefix "left' or "right", it is now the spatial position that is the focus of attention, rather than any other connection or phenotypic variation that may exist. 